\title{
Analysis of Different Vibration Control Strategies for Soft Mounted Induction Motors with Sleeve Bearings Using Active Motor Foot Mounts
}

\section{Ulrich Werner}

Faculty of Electrical Engineering, Precision Engineering, Information Technology, Georg Simon Ohm University of Applied Sciences Nuremberg, Nuremberg, Germany

Email: ulrich.werner@th-nuernberg.de

How to cite this paper: Werner, U. (2019) Analysis of Different Vibration Control Strategies for Soft Mounted Induction Motors with Sleeve Bearings Using Active Motor Foot Mounts. Journal of Applied Mathematics and Physics, 7, 611-637. https://doi.org/10.4236/jamp.2019.73045

Received: February 26, 2019

Accepted: March 22, 2019

Published: March 25, 2019

Copyright $\odot 2019$ by author(s) and Scientific Research Publishing Inc. This work is licensed under the Creative Commons Attribution International License (CC BY 4.0).

http://creativecommons.org/licenses/by/4.0/

\begin{abstract}
The paper presents a theoretical analysis of different vibration control strategies of soft mounted induction motors with sleeve bearings, using active motor foot mounts. After the vibration model is presented, different controllers in combination with different feedback strategies are mathematically investigated. The focus is here on the forced vibrations, caused by dynamic rotor eccentricity-rotor mass eccentricity, magnetic eccentricity and bent rotor deflection. After the mathematically coherences are described, a numerical example is shown, where the forced vibrations caused by bent rotor deflection are investigated, for different control strategies, where the mass matrix, the stiffness matrix and the damping matrix are influenced by different control parameters. The aim of the paper is to show the mathematically coherences and the possibility to influence the vibration behaviour, by different control strategies to optimize the vibration behaviour of soft mounted induction motors.
\end{abstract}

\section{Keywords}

Active Vibration Control, Control Strategies, Induction Motor, Soft

Foundation, Motor Foot Mounts

\section{Introduction}

In praxis, large induction motors (rated power $>1 \mathrm{MW}$ ) are often mounted on elastic steel frame foundations. Due to the elasticity of the foundation, the vibra- 
tion behaviour is mostly completely different to the vibration behaviour in the test field of the motor supplier, where this kind of motors is mostly mounted on a massive foundation, referring to the standard IEC 60034-14 [1]. The result is that critical speeds will occur in the operation speed range, which may lead to vibration problems [2] [3] [4] [5]. Therefore, the idea is to use active vibration control, which is a very powerful instrument, to optimize the vibration behaviour [6] [7] [8] [9]. For rotating machinery sometimes active magnetic bearings are used, especially for high speed applications, and have been optimized during time [10] [11] [12] [13] [14]. But also actuators-used as active mounts-have been investigated, to optimize the vibration behaviour of rotating machinery [15] [16] [17] [18] [19]. The idea is now to use active motor foot mounts between the feet of large induction motors and soft steel frame foundations, to optimize the vibration behaviour of the induction motors on such soft foundations, which has been basically described in [20], but only for a special controller, an ideal proportional-derivative-controller (PD-controller), and only for a feedback of the vertical motor feet displacements and only for fixed controller parameters.

The aim of the paper is now to analyze the influence of different controller structures in combination with different feedback strategies and different controller parameters on the vibration quality of the motor. Therefore different feedbacks of the motor feet movement-displacements, velocities and accelerations-are here investigated in conjunction with different controller structures, e.g. a proportional-controller (P-controller), integral-controller (I-controller), proportional-integral-controller (PI-controller), proportional-derivative-controller (PD-controller) and proportional-integral-derivative controller (PID-controller). The combinations of controllers and feedbacks are chosen in such a manner, that the controller parameters can be directly implemented into the system matrices-the mass matrix, stiffness matrix and damping matrix. The reason is, that the excitations, which are here considered-rotor mass eccentricity, magnetic eccentricity and bent rotor deflection-are depending on the rotor angular frequency $\Omega$, as well as some system parameters, which occur in the system matrices, e.g. electromagnetic stiffness and damping coefficients of the induction motor [20]-[28], oil film stiffness and damping coefficients of the sleeve bearings [29] [30] [31] [32], and the mechanical damping coefficients of the system-described by the corresponding loss factor, stiffness and the rotor angular frequency $\Omega$-e.g. damping of the rotor, of the bearing housings with end-shields, of the elastic steel frame foundation and of the actuators. If now also the controller parameters can be integrated into the system matrices, the differential equation system will remain linear and a simple solution will be possible. The other reason for this procedure is, that it can be shown, where the control parameters occur in each matrix, which is very interesting, when analyzing the vibration behaviour for different control strategies. Of course other control strategies with special controller structures are possible, but are not considered here in this paper. 


\section{Vibration Model}

The vibration model is a simplified plane multibody model (Figure 1), generally based on [20]. However the huge enhancement in this paper is, that in contrast to [20], where only the displacements of the motor feet have been led back to ideal PD-controllers, here also the velocities and accelerations of the motor feet will be lead back to different controllers, which will be P/I/PI/PD and PID-controllers. All this different combinations of feedbacks and controller structure will be analyzed and discussed. Additionally to [20], also not only particular control parameters will be analyzed but also the influence of a certain range of the control parameters on the vibration behaviour will be analyzed.

The model (Figure 1) contains two main masses, the rotor mass $m_{\mathrm{w}}$ and the stator mass $m_{s}$, with the inertia $\theta_{\mathrm{sx}}$. Additional masses are the mass of each shaft journal $m_{\mathrm{v}}$ and of each bearing housing $m_{\mathrm{b}}$ for each rotor side, as well as the masses of the actuators $m_{\text {as }}$ (Stator) and $m_{\text {aa }}$ (armature) and the foundation masses $m_{\mathrm{fL}}$ and $m_{\mathrm{fR}}$ for each motor side. The rotation of the rotor is described by the rotary angular frequency $\Omega$. The rotor has the stiffness $c$ and damping $d_{\mathrm{i}}$ (rotating damping) and is linked to the sleeve bearing housings by the stiffness and the damping matrices $\boldsymbol{C}_{\mathrm{v}}$ and $\boldsymbol{D}_{\mathrm{v}}$ of the oil film. The stiffness and damping matrix $\boldsymbol{C}_{\mathrm{b}}$ and $\boldsymbol{D}_{\mathrm{b}}$ of the sleeve bearing housings with end-shields, link the sleeve bearing housings to the stator housing.

The influence of the electromagnetism in the induction motor, is include by the electromagnetic spring and damper matrix $\boldsymbol{C}_{\mathrm{m}}$ and $\boldsymbol{D}_{\mathrm{m}}$, referring to [20]-[28]. The stator structure can be defined to be rigid, when comparing to the soft steel frame foundation. The stiffness and damping of the actuators is described by the actuator stiffness matrix $C_{\mathrm{a}}$ and the actuator damping matrix $\boldsymbol{D}_{\mathrm{a}}$. The actuator forces are described by $f_{\mathrm{azL}}$ and $f_{\mathrm{azR}}$. All these actuator values are related on one motor side. The stiffness and damping of the foundation is described by the stiffness matrices $\boldsymbol{C}_{\mathrm{fL}}$ and $\boldsymbol{C}_{\mathrm{fR}}$ and the damping matrices $\boldsymbol{D}_{\mathrm{fL}}$ and $\boldsymbol{D}_{\mathrm{fR}}$. For the excitation, three different kinds of dynamic eccentricity are considered here-eccentricity of rotor mass, bent rotor deflection and magnetic eccentricity (Figure 2), which are occurring with rotor angular frequency $\Omega$.

The oil film stiffness and damping coefficients $c_{\mathrm{ij}}=c_{\mathrm{ij}}(\Omega)$ and $d_{\mathrm{ij}}=d_{\mathrm{ij}}(\Omega)$ can be calculated, referring to [29] [30] [31] [32]. The electromagnetic stiffness coefficient $c_{\mathrm{md}}\left(\omega_{\mathrm{F}}\right)$ and damping coefficient $d_{\mathrm{m}}\left(\omega_{\mathrm{F}}\right)$, depending on the harmonic slip $s_{v}$, and therefore depending on the whirling angular frequency $\omega_{\mathrm{F}}$ can be calculated referring to [20]-[28]. All mechanical damping coefficients $d_{\mathrm{n}}\left(d_{\mathrm{i}}, d_{\mathrm{bz}}, d_{\mathrm{by}}, d_{\mathrm{fzL}}, d_{\mathrm{fyL}}, d_{\mathrm{fz} R}, d_{\mathrm{fy} R}, d_{\mathrm{az}}, d_{\mathrm{ay}}\right)$ can be calculated by the corresponding mechanical loss factor $\tan \delta_{\mathrm{n}}$, the corresponding stiffness $c_{\mathrm{n}}$ and by the whirling frequency $\omega_{\mathrm{F}}$, referring to [4] and [20].

$$
d_{\mathrm{n}}=\frac{c_{\mathrm{n}} \cdot \tan \delta_{\mathrm{n}}}{\omega_{\mathrm{F}}}
$$




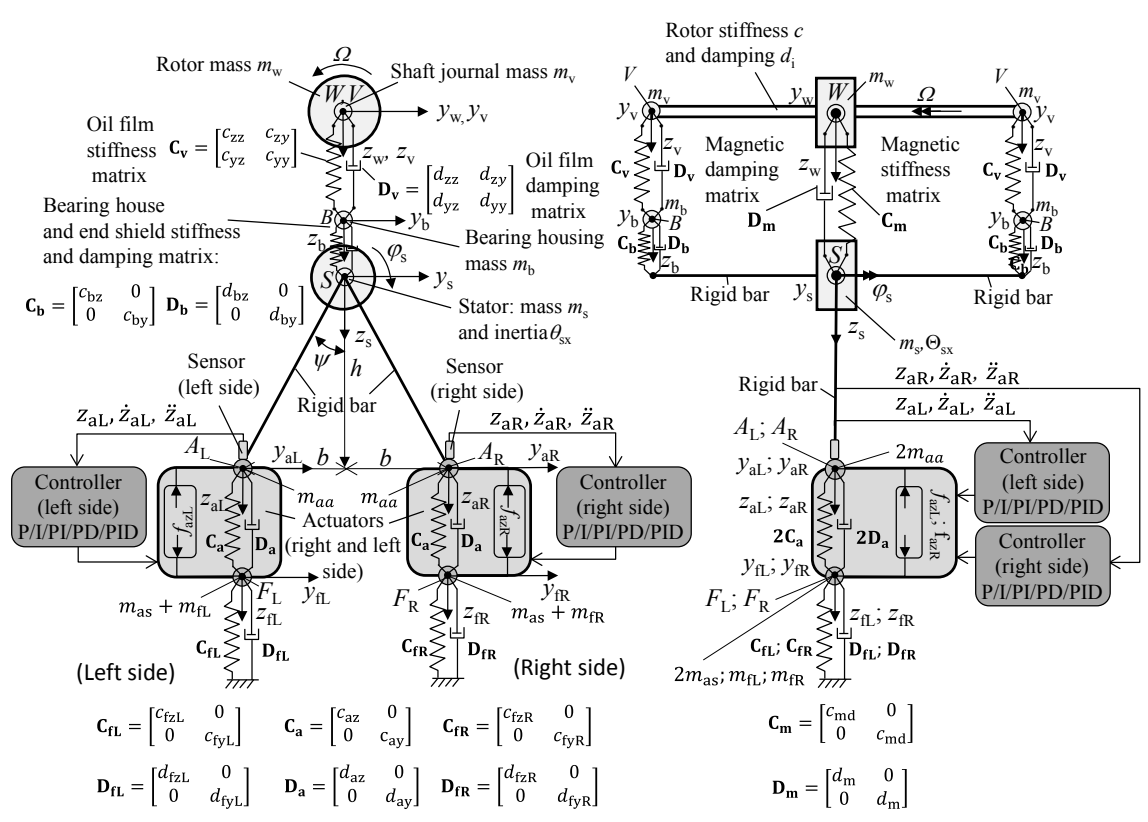

Notice: Magnetic spring constant is not pictured in the front view

Figure 1. Vibration model with different controllers and feedbacks.

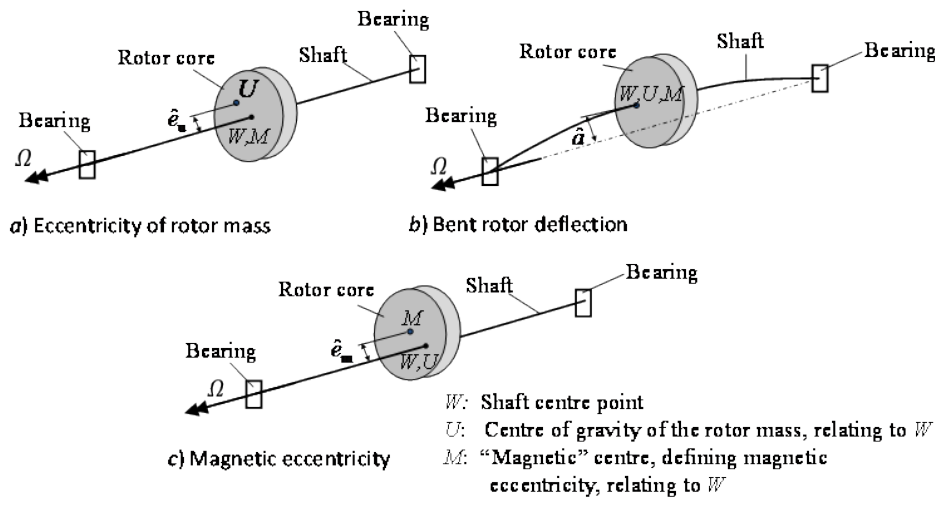

Figure 2. Different excitations by dynamic eccentricity [20] and [28].

For forced vibration due to dynamic eccentricity, the whirling angular frequency is equal to the rotor angular frequency $\omega_{\mathrm{F}}=\Omega$.

\section{Mathematical Model}

\subsection{Open Control Loops}

If the control loops are open, the equations of motions can be described by following differential equation, based on [20] and [28]:

$$
\boldsymbol{M} \cdot \ddot{\boldsymbol{q}}+\boldsymbol{D} \cdot \dot{\boldsymbol{q}}+\boldsymbol{C} \cdot \boldsymbol{q}=\boldsymbol{f}_{\mathrm{e}}
$$

Including following linearization for the motor feet displacements, because of small displacements:

$$
\begin{aligned}
z_{\mathrm{aL}} & =z_{\mathrm{s}}-\varphi_{\mathrm{s}} \cdot b \\
z_{\mathrm{aR}} & =z_{\mathrm{s}}+\varphi_{\mathrm{s}} \cdot b
\end{aligned}
$$




$$
y_{\mathrm{aL}}=y_{\mathrm{aR}}=y_{\mathrm{s}}-\varphi_{\mathrm{s}} \cdot h
$$

With the coordinate vector $q$ :

$$
\boldsymbol{q}=\left[z_{\mathrm{s}} ; z_{\mathrm{w}} ; y_{\mathrm{s}} ; y_{\mathrm{w}} ; \varphi_{\mathrm{s}} ; z_{\mathrm{v}} ; z_{\mathrm{b}} ; z_{\mathrm{fL}} ; z_{\mathrm{fR}} ; y_{\mathrm{v}} ; y_{\mathrm{b}} ; y_{\mathrm{fL}} ; y_{\mathrm{fR}}\right]^{\mathrm{T}}
$$

and with the vector of the excitation forces $\boldsymbol{f}_{\mathrm{e}}$ :

$$
\boldsymbol{f}_{\mathrm{e}}=\hat{\boldsymbol{f}}_{\mathrm{e}} \cdot e^{j \Omega t}=\left(\hat{\boldsymbol{f}}_{\mathrm{e}, \mathrm{u}}+\hat{\boldsymbol{f}}_{\mathrm{e}, \mathrm{m}}+\hat{\boldsymbol{f}}_{\mathrm{e}, \mathrm{a}}\right) \cdot e^{j \Omega t}
$$

This excitation amplitude vector $\hat{\boldsymbol{f}}_{\mathrm{e}}$ is split into following excitation vectors: - Vector $\hat{\boldsymbol{f}}_{\mathrm{e}, \mathrm{u}}$ : It describes the excitation by rotor mass eccentricity, which is e.g. caused by residual unbalance, which remains after the balancing process, with the amplitude $\hat{e}_{\mathrm{u}}$ and the phase $\varphi_{\mathrm{u}}$ of the mass eccentricity:

$$
\hat{\boldsymbol{f}}_{\mathrm{e}, \mathrm{u}}=[0 ; 1 ; 0 ;-j ; 0 ; 0 ; 0 ; 0 ; 0 ; 0 ; 0 ; 0 ; 0]^{\mathrm{T}} \cdot \hat{e}_{\mathrm{u}} \cdot m_{w} \cdot \Omega^{2} \cdot e^{j \varphi_{u}}
$$

- Vector $\hat{\boldsymbol{f}}_{\mathrm{e}, \mathrm{m}}$ : It describes the excitation by magnetic eccentricity, which is e.g. caused by deviation of concentricity between the inner diameter of the rotor core and the outer diameter of the rotor core, with the amplitude $\hat{e}_{\mathrm{m}}$ and the phase $\varphi_{\mathrm{m}}$ of the magnetic eccentricity:

$$
\begin{aligned}
\hat{\boldsymbol{f}}_{\mathrm{e}, \mathrm{m}}= & {\left[-c_{m d}+j \Omega d_{m} ; c_{m d}-j \Omega d_{m} ; j c_{m d}+\Omega d_{m} ;-j c_{m d}-\Omega d_{m}\right.} \\
& 0 ; 0 ; 0 ; 0 ; 0 ; 0 ; 0 ; 0 ; 0]^{\mathrm{T}} \cdot \hat{e}_{\mathrm{m}} \cdot e^{j \varphi_{m}}
\end{aligned}
$$

In $\hat{\boldsymbol{f}}_{\mathrm{e}, \mathrm{m}}$, not only the radial magnetic excitation force $\hat{e}_{\mathrm{m}} \cdot c_{\mathrm{md}}$ is considered, but also the tangential magnetic excitation force $\hat{e}_{\mathrm{m}} \cdot \Omega \cdot d_{\mathrm{m}}$, which was shown in [28], but was neglected there as a simplification.

- Vector $\hat{\boldsymbol{f}}_{\mathrm{e}, \mathrm{a}}$ : It describes the excitation by bent rotor deflection, which is e.g. caused by thermal bending of the rotor, with the amplitude $\hat{a}$ and the phase $\varphi_{\mathrm{a}}$ of the bent rotor deflection:

$$
\hat{\boldsymbol{f}}_{\mathrm{e}, \mathrm{a}}=[0 ; 1 ; 0 ;-j ; 0 ;-1 ; 0 ; 0 ; 0 ; j ; 0 ; 0 ; 0]^{\mathrm{T}} \cdot \hat{a} \cdot c \cdot e^{j \varphi_{a}}
$$

The mass matrix $\boldsymbol{M}$ is described by: (11)

$$
\boldsymbol{M}=\left[\begin{array}{ccccc}
m_{\mathrm{s}}+2 m_{\mathrm{aa}} & 0 & 0 & 0 & 0 \\
0 & m_{\mathrm{w}} & 0 & 0 & 0 \\
0 & 0 & m_{\mathrm{s}}+2 m_{\mathrm{aa}} & 0 & -2 m_{\mathrm{aa}} \cdot h \\
0 & 0 & 0 & m_{\mathrm{w}} & 0 \\
0 & 0 & -2 m_{\mathrm{aa}} \cdot h & 0 & \theta_{s x}+2 m_{a a}\left(b^{2}+h^{2}\right) \\
0 & 0 & 0 & 0 & 0 \\
0 & 0 & 0 & 0 & 0 \\
0 & 0 & 0 & 0 & 0 \\
0 & 0 & 0 & 0 & 0 \\
0 & 0 & 0 & 0 & 0 \\
0 & 0 & 0 & 0 & 0 \\
0 & 0 & 0 & 0 & 0 \\
0 & 0 & 0 & 0 & 0
\end{array}\right.
$$




$\left.\begin{array}{cccccccc}0 & 0 & 0 & 0 & 0 & 0 & 0 & 0 \\ 0 & 0 & 0 & 0 & 0 & 0 & 0 & 0 \\ 0 & 0 & 0 & 0 & 0 & 0 & 0 & 0 \\ 0 & 0 & 0 & 0 & 0 & 0 & 0 & 0 \\ 0 & 0 & 0 & 0 & 0 & 0 & 0 & 0 \\ 2 m_{\mathrm{v}} & 0 & 0 & 0 & 0 & 0 & 0 & 0 \\ 0 & 2 m_{\mathrm{b}} & 0 & 0 & 0 & 0 & 0 & 0 \\ 0 & 0 & m_{\mathrm{as}}+m_{\mathrm{fL}} & 0 & 0 & 0 & 0 & 0 \\ 0 & 0 & 0 & m_{\mathrm{as}}+m_{\mathrm{fR}} & 0 & 0 & 0 & 0 \\ 0 & 0 & 0 & 0 & 2 m_{\mathrm{v}} & 0 & 0 & 0 \\ 0 & 0 & 0 & 0 & 0 & 2 m_{\mathrm{b}} & 0 & 0 \\ 0 & 0 & 0 & 0 & 0 & 0 & m_{\mathrm{as}}+m_{\mathrm{fL}} & 0 \\ 0 & 0 & 0 & 0 & 0 & 0 & 0 & m_{\mathrm{as}}+m_{\mathrm{fR}}\end{array}\right]$

The damping matrix $\boldsymbol{D}$ is described by: (12)

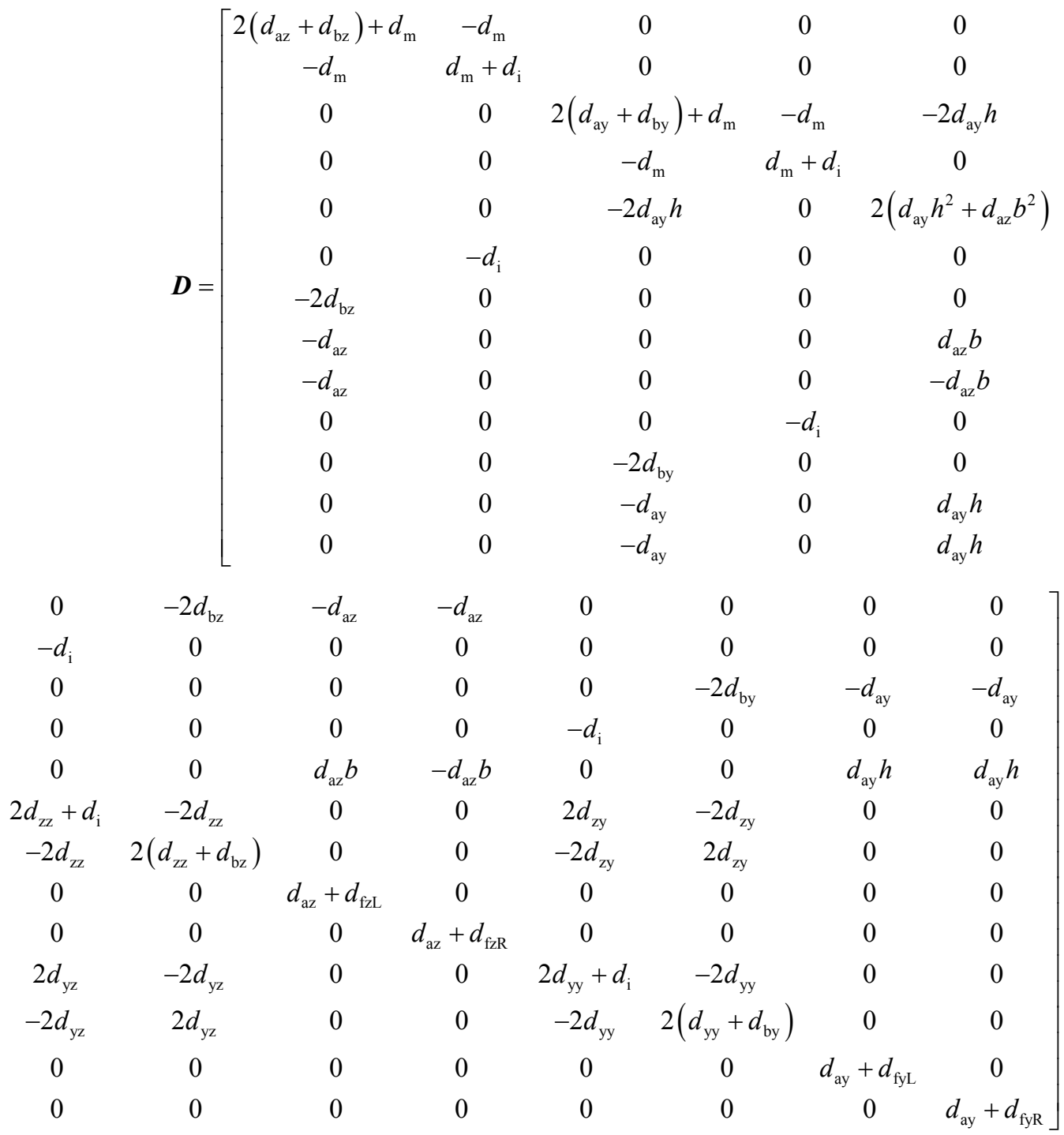

The stiffness matrix $\boldsymbol{C}$ is described by: (13) 


\begin{tabular}{|c|c|c|c|c|c|c|c|}
\hline & & $\left(c_{\mathrm{az}}+c_{\mathrm{bz}}\right)-c_{\mathrm{md}}$ & $c_{\mathrm{md}}$ & 0 & & 0 & 0 \\
\hline & & $c_{\mathrm{md}}$ & $c-c_{\mathrm{md}}$ & 0 & & $d_{\mathrm{i}} \Omega$ & 0 \\
\hline & & 0 & 0 & $2\left(c_{\text {ay }}+c_{\text {by }}\right)$ & )$-c_{\mathrm{md}}$ & $c_{\mathrm{md}}$ & $-2 c_{\mathrm{ay}} h$ \\
\hline & & 0 & $-d_{\mathrm{i}} \Omega$ & $c_{\mathrm{md}}$ & & $-c_{\mathrm{md}}$ & 0 \\
\hline & & 0 & 0 & $-2 c_{\text {ay }} /$ & & $2\left(c_{\text {ay }} h\right.$ & $\left.h^{2}+c_{\mathrm{az}} b^{2}\right)$ \\
\hline & & 0 & $-c$ & 0 & & $-d_{\mathrm{i}} \Omega$ & 0 \\
\hline & $C=$ & $-2 c_{\mathrm{bz}}$ & 0 & 0 & & 0 & 0 \\
\hline & & $-c_{\mathrm{az}}$ & 0 & 0 & & 0 & $c_{\mathrm{az}} b$ \\
\hline & & $-c_{\mathrm{az}}$ & 0 & 0 & & 0 & $-c_{\mathrm{az}} b$ \\
\hline & & 0 & $d_{\mathrm{i}} \Omega$ & 0 & & $-c$ & 0 \\
\hline & & 0 & 0 & $-2 c_{\text {by }}$ & & 0 & 0 \\
\hline & & 0 & 0 & $-c_{\mathrm{ay}}$ & & 0 & $c_{\mathrm{ay}} h$ \\
\hline & L & 0 & 0 & $-c_{\mathrm{ay}}$ & & 0 & $c_{\text {ay }} h$ \\
\hline 0 & $-2 c_{\mathrm{bz}}$ & $-c_{\mathrm{az}}$ & $-c_{\mathrm{az}}$ & 0 & 0 & 0 & 0 \\
\hline$-c$ & 0 & 0 & 0 & $-d_{\mathrm{i}} \Omega$ & 0 & 0 & 0 \\
\hline 0 & 0 & 0 & 0 & 0 & $-2 c_{\text {by }}$ & $-c_{\text {ay }}$ & $-c_{\text {ay }}$ \\
\hline$d_{\mathrm{i}} \Omega$ & 0 & 0 & 0 & $-c$ & 0 & 0 & 0 \\
\hline 0 & 0 & $c_{\mathrm{az}} b$ & $-c_{\mathrm{az}} b$ & 0 & 0 & $c_{\text {ay }} h$ & $c_{\mathrm{ay}} h$ \\
\hline $2 c_{\mathrm{zz}}+c$ & $-2 c_{\mathrm{zz}}$ & 0 & 0 & $2 c_{z y}+d_{\mathrm{i}} \Omega$ & $-2 c_{\mathrm{zy}}$ & 0 & 0 \\
\hline$-2 c_{\mathrm{zz}}$ & $2\left(c_{\mathrm{zz}}+c_{\mathrm{bz}}\right)$ & 0 & 0 & $-2 c_{\mathrm{zy}}$ & $2 c_{\mathrm{zy}}$ & 0 & 0 \\
\hline 0 & 0 & $c_{\mathrm{az}}+c_{\mathrm{fzL}}$ & 0 & 0 & 0 & 0 & 0 \\
\hline 0 & 0 & 0 & $c_{\mathrm{az}}+c_{\mathrm{fz} \mathrm{R}}$ & 0 & 0 & 0 & 0 \\
\hline $2 c_{\mathrm{yz}}-d_{\mathrm{i}} \Omega$ & $-2 c_{\mathrm{yz}}$ & 0 & 0 & $2 c_{\mathrm{yy}}+c$ & $-2 c_{\mathrm{yy}}$ & 0 & 0 \\
\hline$-2 c_{\mathrm{yz}}$ & $2 c_{\mathrm{yz}}$ & 0 & 0 & $-2 c_{\mathrm{yy}}$ & $2\left(c_{\mathrm{yy}}+c_{\mathrm{by}}\right)$ & 0 & 0 \\
\hline 0 & 0 & 0 & 0 & 0 & 0 & $c_{\mathrm{ay}}+c_{\mathrm{fyL}}$ & 0 \\
\hline 0 & 0 & 0 & 0 & 0 & 0 & 0 & $c_{\text {ay }}+c_{\text {fyR }}$ \\
\hline
\end{tabular}

\subsection{Closed Control Loops}

If the control loops are closed, the actuator force vector $f_{\mathrm{a}}$ has to be implemented into the differential equation, so that it becomes:

$$
\boldsymbol{M} \cdot \ddot{\boldsymbol{q}}+\boldsymbol{D} \cdot \dot{\boldsymbol{q}}+\boldsymbol{C} \cdot \boldsymbol{q}=\boldsymbol{f}_{\mathrm{e}}+\boldsymbol{f}_{\mathrm{a}}
$$

The actuator force vector can be split into the actuator force vector on the left side $\boldsymbol{f}_{\mathrm{azL}}$ and on the right side $\boldsymbol{f}_{\mathrm{azR}}$ of the motor:

$$
\boldsymbol{f}_{\mathrm{a}}=\boldsymbol{f}_{\mathrm{azL}}+\boldsymbol{f}_{\mathrm{azR}}=\boldsymbol{P}_{\mathrm{azL}} \cdot f_{\mathrm{azL}}+\boldsymbol{P}_{\mathrm{azR}} \cdot f_{\mathrm{azR}}
$$

with: $\quad \boldsymbol{P}_{\mathrm{azL}}=[1 ; 0 ; 0 ; 0 ;-b ; 0 ; 0 ;-1 ; 0 ; 0 ; 0 ; 0 ; 0]^{\mathrm{T}}$

$$
\boldsymbol{P}_{\mathrm{azR}}=[1 ; 0 ; 0 ; 0 ; b ; 0 ; 0 ; 0 ;-1 ; 0 ; 0 ; 0 ; 0]^{\mathrm{T}}
$$


The actuator forces for the left side $f_{\mathrm{azL}}$ and for the right side $f_{\mathrm{azR}}$ are depending on the control strategy and the values, which are lead back to the controllers. Therefore, they are a function of the motor feet displacements, velocities and accelerations.

$$
f_{\mathrm{azL}}=f_{\mathrm{azL}}\left(z_{\mathrm{aL}}, \dot{z}_{\mathrm{aL}}, \ddot{z}_{\mathrm{aL}}\right) ; f_{\mathrm{azR}}=f_{\mathrm{azR}}\left(z_{\mathrm{aR}}, \dot{z}_{\mathrm{aR}}, \ddot{z}_{\mathrm{aR}}\right)
$$

With the cinematic constraints follows:

$$
f_{\mathrm{azL}}=f_{\mathrm{azL}}\left(z_{\mathrm{s}}, \varphi_{s}, \dot{z}_{\mathrm{s}}, \dot{\varphi}_{s}, \ddot{z}_{s}, \ddot{\varphi}_{s}\right) ; f_{\mathrm{azR}}=f_{\mathrm{azR}}\left(z_{\mathrm{s}}, \varphi_{s}, \dot{z}_{\mathrm{s}}, \dot{\varphi}_{s}, \ddot{z}_{s}, \ddot{\varphi}_{s}\right)
$$

\subsubsection{Feedback of the Motor Feet Displacements}

\section{P-controllers:}

Using P-controllers-with the control parameters $K_{\mathrm{PL}}$ and $K_{\mathrm{PR}}$ for the left side and for the right side of the motor-with negative feedback loops for the vertical motor feet displacements $z_{\mathrm{aL}}$ and $z_{\mathrm{aR}}$, the actuator forces can described by:

$$
\boldsymbol{f}_{\mathrm{a}}=-\boldsymbol{P}_{\mathrm{azL}} \cdot K_{\mathrm{PL}} \cdot z_{\mathrm{aL}}-\boldsymbol{P}_{\mathrm{azR}} \cdot K_{\mathrm{PR}} \cdot z_{\mathrm{aR}}
$$

With the cinematic constrains follows:

$$
\boldsymbol{f}_{\mathrm{a}}=-\boldsymbol{P}_{\mathrm{azL}} \cdot K_{\mathrm{PL}} \cdot\left(z_{\mathrm{s}}-\varphi_{\mathrm{s}} \cdot b\right)-\boldsymbol{P}_{\mathrm{azR}} \cdot K_{\mathrm{PR}} \cdot\left(z_{\mathrm{s}}+\varphi_{\mathrm{s}} \cdot b\right)
$$

Therefore, the differential equation system can now be described by:

$$
\begin{aligned}
& \boldsymbol{M} \cdot \ddot{\boldsymbol{q}}+\boldsymbol{D} \cdot \dot{\boldsymbol{q}}+\boldsymbol{C} \cdot \boldsymbol{q}+\boldsymbol{P}_{\mathrm{azL}} \cdot K_{\mathrm{PL}} \cdot\left(z_{\mathrm{s}}-\varphi_{\mathrm{s}} \cdot b\right) \\
& +\boldsymbol{P}_{\mathrm{azR}} \cdot K_{\mathrm{PR}} \cdot\left(z_{\mathrm{s}}+\varphi_{\mathrm{s}} \cdot b\right)=\boldsymbol{f}_{\mathrm{e}}
\end{aligned}
$$

The control parameters $K_{\mathrm{PL}}, K_{\mathrm{PR}}$ can now be integrated into the stiffness matrix leading to a controlled system stiffness matrix $\boldsymbol{C}_{\mathrm{KP}}$. The new differential equation system can now be described by:

$$
\boldsymbol{M} \cdot \ddot{\boldsymbol{q}}+\boldsymbol{D} \cdot \dot{\boldsymbol{q}}+\boldsymbol{C}_{\mathrm{KP}} \cdot \boldsymbol{q}=\boldsymbol{f}_{\mathrm{e}}
$$

With stiffness matrix $C_{\mathrm{KP}}:(24)$

$\boldsymbol{C}_{\mathrm{KP}}=\left[\begin{array}{ccccc}2\left(c_{\mathrm{az}}+c_{\mathrm{bz}}\right)-c_{\mathrm{md}}+K_{\mathrm{PR}}+K_{\mathrm{PL}} & c_{\mathrm{md}} & 0 & 0 & b\left(K_{\mathrm{PR}}-K_{\mathrm{PL}}\right) \\ c_{\mathrm{md}} & c-c_{\mathrm{md}} & 0 & d_{\mathrm{i}} \Omega & 0 \\ 0 & 0 & 2\left(c_{\mathrm{ay}}+c_{\mathrm{by}}\right)-c_{\mathrm{md}} & c_{\mathrm{md}} & -2 c_{\mathrm{ay}} h \\ 0 & -d_{\mathrm{i}} \Omega & c_{\mathrm{md}} & c-c_{\mathrm{md}} & 0 \\ b\left(K_{\mathrm{PR}}-K_{\mathrm{PL}}\right) & 0 & -2 c_{\mathrm{ay}} h & 0 & 2\left(c_{\mathrm{ay}} h^{2}+c_{\mathrm{az}} b^{2}\right)+b^{2}\left(K_{\mathrm{PL}}+K_{\mathrm{PR}}\right) \\ 0 & -c & 0 & -d_{\mathrm{i}} \Omega & 0 \\ -2 c_{\mathrm{bz}} & 0 & 0 & 0 & 0 \\ -c_{\mathrm{az}}-K_{\mathrm{PL}} & 0 & 0 & 0 & c_{\mathrm{az}} b+K_{\mathrm{PL}} b \\ -c_{\mathrm{az}}-K_{\mathrm{PR}} & 0 & 0 & -c_{\mathrm{az}} b-K_{\mathrm{PR}} b \\ 0 & d_{\mathrm{i}} \Omega & 0 & -c & 0 \\ 0 & 0 & -2 c_{\mathrm{by}} & 0 & 0 \\ 0 & 0 & -c_{\mathrm{ay}} & 0 & c_{\mathrm{ay}} h \\ 0 & 0 & -c_{\mathrm{ay}} & 0 & c_{\mathrm{ay}} h\end{array}\right.$




$\left.\begin{array}{cccccccc}0 & -2 c_{\mathrm{bz}} & -c_{\mathrm{az}} & -c_{\mathrm{az}} & 0 & 0 & 0 & 0 \\ -c & 0 & 0 & 0 & -d_{\mathrm{i}} \Omega & 0 & 0 & 0 \\ 0 & 0 & 0 & 0 & 0 & -2 c_{\mathrm{by}} & -c_{\mathrm{ay}} & -c_{\mathrm{ay}} \\ d_{\mathrm{i}} \Omega & 0 & 0 & 0 & -c & 0 & 0 & 0 \\ 0 & 0 & c_{\mathrm{az}} b & -c_{\mathrm{az}} b & 0 & 0 & c_{\mathrm{ay}} h & c_{\mathrm{ay}} h \\ 2 c_{\mathrm{zz}}+c & -2 c_{\mathrm{zz}} & 0 & 0 & 2 c_{\mathrm{zy}}+d_{\mathrm{i}} \Omega & -2 c_{\mathrm{zy}} & 0 & 0 \\ -2 c_{\mathrm{zz}} & 2\left(c_{\mathrm{zz}}+c_{\mathrm{bz}}\right) & 0 & 0 & -2 c_{\mathrm{zy}} & 2 c_{\mathrm{zy}} & 0 & 0 \\ 0 & 0 & c_{\mathrm{az}}+c_{\mathrm{fzL}} & 0 & 0 & 0 & 0 & 0 \\ 0 & 0 & 0 & c_{\mathrm{az}}+c_{\mathrm{fz} \mathrm{R}} & 0 & 0 & 0 & 0 \\ 2 c_{\mathrm{yz}}-d_{\mathrm{i}} \Omega & -2 c_{\mathrm{yz}} & 0 & 0 & 2 c_{\mathrm{yy}}+c & -2 c_{\mathrm{yy}} & 0 & 0 \\ -2 c_{\mathrm{yz}} & 2 c_{\mathrm{yz}} & 0 & 0 & -2 c_{\mathrm{yy}} & 2\left(c_{\mathrm{yy}}+c_{\mathrm{by}}\right) & 0 & 0 \\ 0 & 0 & 0 & 0 & 0 & 0 & c_{\mathrm{ay}}+c_{\mathrm{fyL}} & 0 \\ 0 & 0 & 0 & 0 & 0 & 0 & 0 & c_{\mathrm{ay}}+c_{\mathrm{fy} \mathrm{R}}\end{array}\right]$

\section{PD-controllers:}

For ideal PD-controllers-with the control parameters $K_{\mathrm{PL}}, K_{\mathrm{PR}}$ and $K_{\mathrm{DL}}$, $K_{\mathrm{DR}}$ for the left side and for the right side of the motor-with negative feedback loops for the vertical motor feet displacements $z_{\mathrm{aL}}$ and $z_{\mathrm{aR}}$, the actuator forces become:

$$
\begin{aligned}
\boldsymbol{f}_{\mathrm{a}}= & -\boldsymbol{P}_{\mathrm{azL}} \cdot\left(K_{\mathrm{PL}} \cdot z_{\mathrm{aL}}+K_{\mathrm{DL}} \cdot \frac{\mathrm{d} z_{\mathrm{aL}}}{\mathrm{d} t}\right) \\
& -\boldsymbol{P}_{\mathrm{azR}} \cdot\left(K_{\mathrm{PR}} \cdot z_{\mathrm{aR}}+K_{\mathrm{DR}} \cdot \frac{\mathrm{d} z_{\mathrm{aR}}}{\mathrm{d} t}\right)
\end{aligned}
$$

With the cinematic constrains follows:

$$
\begin{aligned}
\boldsymbol{f}_{\mathrm{a}}= & -\boldsymbol{P}_{\mathrm{azL}} \cdot\left(K_{\mathrm{PL}} \cdot\left(z_{\mathrm{s}}-\varphi_{\mathrm{s}} \cdot b\right)+K_{\mathrm{DL}} \cdot\left(\dot{z}_{\mathrm{s}}-\dot{\varphi}_{\mathrm{s}} \cdot b\right)\right) \\
& -\boldsymbol{P}_{\mathrm{azR}} \cdot\left(K_{\mathrm{PR}} \cdot\left(z_{\mathrm{s}}+\varphi_{\mathrm{s}} \cdot b\right)+K_{\mathrm{DR}} \cdot\left(\dot{z}_{\mathrm{s}}+\dot{\varphi}_{\mathrm{s}} \cdot b\right)\right)
\end{aligned}
$$

Therefore, the differential equation system can now be described by:

$$
\begin{aligned}
& \boldsymbol{M} \cdot \ddot{\boldsymbol{q}}+\boldsymbol{D} \cdot \dot{\boldsymbol{q}}+\boldsymbol{C} \cdot \boldsymbol{q} \\
& +\boldsymbol{P}_{\mathrm{azL}} \cdot\left(K_{\mathrm{PL}} \cdot\left(z_{\mathrm{s}}-\varphi_{\mathrm{s}} \cdot b\right)+K_{\mathrm{DL}} \cdot\left(\dot{z}_{\mathrm{s}}-\dot{\varphi}_{\mathrm{s}} \cdot b\right)\right) \\
& +\boldsymbol{P}_{\mathrm{azR}} \cdot\left(K_{\mathrm{PR}} \cdot\left(z_{\mathrm{s}}+\varphi_{\mathrm{s}} \cdot b\right)+K_{\mathrm{DR}} \cdot\left(\dot{z}_{\mathrm{s}}+\dot{\varphi}_{\mathrm{s}} \cdot b\right)\right)=\boldsymbol{f}_{\mathrm{e}}
\end{aligned}
$$

The control parameters $K_{\mathrm{PL}}, K_{\mathrm{PR}}$ and $K_{\mathrm{DL}}, K_{\mathrm{DR}}$ can now be integrated into the stiffness matrix and into the damping matrix leading to a controlled system stiffness matrix $\boldsymbol{C}_{\mathrm{KP}}$ and a controlled damping matrix $\boldsymbol{D}_{\mathrm{KD}}$. The new differential equation system can now be described by:

$$
\boldsymbol{M} \cdot \ddot{\boldsymbol{q}}+\boldsymbol{D}_{\mathrm{KD}} \cdot \dot{\boldsymbol{q}}+\boldsymbol{C}_{\mathrm{KP}} \cdot \boldsymbol{q}=\boldsymbol{f}_{\mathrm{e}}
$$

The stiffness matrix $\boldsymbol{C}_{\mathrm{KP}}$ is shown before.

The damping matrix $\boldsymbol{D}_{\mathrm{KD}}$ is described by: (29) 


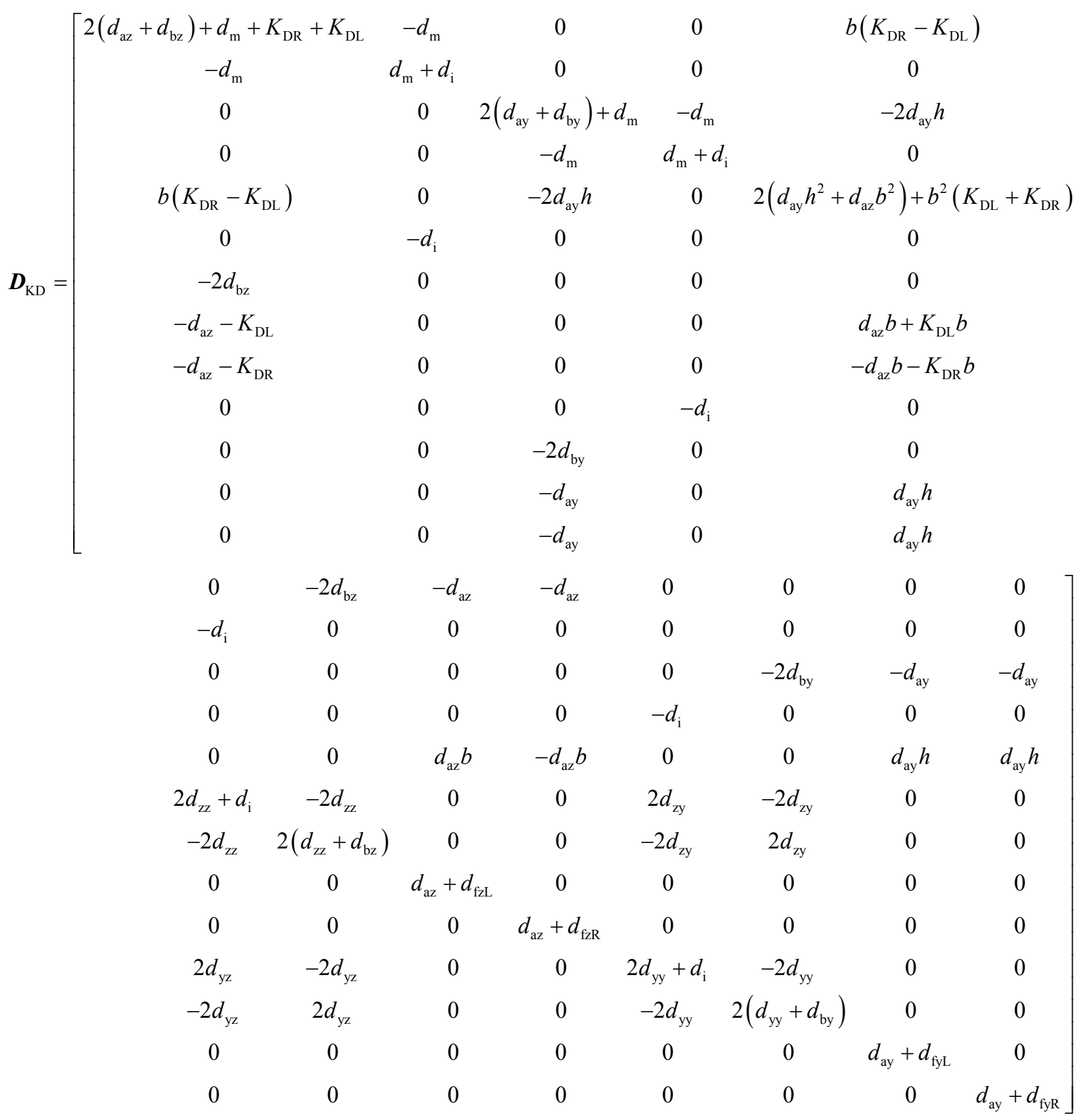

\subsubsection{Feedback of the Motor Feet Velocities}

\section{P-controllers:}

Using P-controllers-with the control parameters $K_{\mathrm{PL}}$ and $K_{\mathrm{PR}}$ for the left side and for the right side of the motor-with negative feedback loops for the vertical motor feet velocities $\dot{z}_{\mathrm{aL}}$ and $\dot{z}_{\mathrm{aR}}$, the actuator forces can described by:

$$
\boldsymbol{f}_{\mathrm{a}}=-\boldsymbol{P}_{\mathrm{azL}} \cdot K_{\mathrm{PL}} \cdot \dot{z}_{\mathrm{aL}}-\boldsymbol{P}_{\mathrm{azR}} \cdot K_{\mathrm{PR}} \cdot \dot{z}_{\mathrm{aR}}
$$

With the cinematic constrains follows the differential equation system:

$$
\boldsymbol{M} \cdot \ddot{\boldsymbol{q}}+\boldsymbol{D} \cdot \dot{\boldsymbol{q}}+\boldsymbol{C} \cdot \boldsymbol{q}+\boldsymbol{P}_{\mathrm{azL}} \cdot K_{\mathrm{PL}} \cdot\left(\dot{z}_{\mathrm{s}}-\dot{\varphi}_{\mathrm{s}} \cdot b\right)+\boldsymbol{P}_{\mathrm{azR}} \cdot K_{\mathrm{PR}} \cdot\left(\dot{z}_{\mathrm{s}}+\dot{\varphi}_{\mathrm{s}} \cdot b\right)=\boldsymbol{f}_{\mathrm{e}}
$$

The control parameters $K_{\mathrm{PL}}, K_{\mathrm{PR}}$ can now be integrated into the damping matrix leading to a controlled damping matrix $\boldsymbol{D}_{\mathrm{KP}}$. The new differential equa- 
tion system can now be described by:

$$
\boldsymbol{M} \cdot \ddot{\boldsymbol{q}}+\boldsymbol{D}_{\mathrm{KP}} \cdot \dot{\boldsymbol{q}}+\boldsymbol{C} \cdot \boldsymbol{q}=\boldsymbol{f}_{\mathrm{e}}
$$

The damping matrix $\boldsymbol{D}_{\mathrm{KP}}$ is identical to $\boldsymbol{D}_{\mathrm{KD}}$ regarding the structure, only the controller parameters have to be replaced:

$$
\boldsymbol{D}_{\mathrm{KD}} \stackrel{K_{\mathrm{DL}} \rightarrow K_{\mathrm{PL}}}{\stackrel{K_{\mathrm{DR}} \rightarrow K_{\mathrm{PR}}}{\longrightarrow}} \boldsymbol{D}_{\mathrm{KP}}
$$

\section{PD-controllers:}

For ideal PD-controllers-with the control parameters $K_{\mathrm{PL}}, K_{\mathrm{PR}}$ and $K_{\mathrm{DL}}$, $K_{\mathrm{DR}}$ for the left side and for the right side of the motor-with negative feedback loops for the vertical motor feet velocities $\dot{z}_{\mathrm{aL}}$ and $\dot{z}_{\mathrm{aR}}$, the actuator forces become:

$$
\boldsymbol{f}_{\mathrm{a}}=-\boldsymbol{P}_{\mathrm{azL}} \cdot\left(K_{\mathrm{PL}} \cdot \dot{z}_{\mathrm{aL}}+K_{\mathrm{DL}} \cdot \frac{\mathrm{d} \dot{z}_{\mathrm{aL}}}{\mathrm{d} t}\right)-\boldsymbol{P}_{\mathrm{azR}} \cdot\left(K_{\mathrm{PR}} \cdot \dot{z}_{\mathrm{aR}}+K_{\mathrm{DR}} \cdot \frac{\mathrm{d} \dot{z}_{\mathrm{aR}}}{\mathrm{d} t}\right)
$$

The differential equation system can now be written by:

$$
\begin{aligned}
& \boldsymbol{M} \cdot \ddot{\boldsymbol{q}}+\boldsymbol{D} \cdot \dot{\boldsymbol{q}}+\boldsymbol{C} \cdot \boldsymbol{q} \\
& +\boldsymbol{P}_{\mathrm{azL}} \cdot\left(K_{\mathrm{PL}} \cdot\left(\dot{z}_{\mathrm{s}}-\dot{\varphi}_{\mathrm{s}} \cdot b\right)+K_{\mathrm{DL}} \cdot\left(\ddot{z}_{\mathrm{s}}-\ddot{\varphi}_{\mathrm{s}} \cdot b\right)\right) \\
& +\boldsymbol{P}_{\mathrm{azR}} \cdot\left(K_{\mathrm{PR}} \cdot\left(\dot{z}_{\mathrm{s}}+\dot{\varphi}_{\mathrm{s}} \cdot b\right)+K_{\mathrm{DR}} \cdot\left(\ddot{z}_{\mathrm{s}}+\ddot{\varphi}_{\mathrm{s}} \cdot b\right)\right)=\boldsymbol{f}_{\mathrm{e}}
\end{aligned}
$$

The control parameters $K_{\mathrm{PL}}, K_{\mathrm{PR}}$ and $K_{\mathrm{DL}}, K_{\mathrm{DR}}$ can be integrated into the damping matrix and into the mass matrix leading to a controlled system damping matrix $\boldsymbol{D}_{\mathrm{KP}}$ and a controlled mass matrix $\boldsymbol{M}_{\mathrm{KD}}$. The new differential equation system becomes:

$$
\boldsymbol{M}_{\mathrm{KD}} \cdot \ddot{\boldsymbol{q}}+\boldsymbol{D}_{\mathrm{KP}} \cdot \dot{\boldsymbol{q}}+\boldsymbol{C} \cdot \boldsymbol{q}=\boldsymbol{f}_{\mathrm{e}}
$$

The damping matrix $\boldsymbol{D}_{\mathrm{KP}}$ was described before. The mass matrix $\boldsymbol{M}_{\mathrm{KD}}$ is: (37)

$$
\boldsymbol{M}_{\mathrm{KD}}=\left[\begin{array}{ccccc}
m_{\mathrm{s}}+2 m_{\mathrm{aa}}+K_{\mathrm{DL}}+K_{\mathrm{DR}} & 0 & 0 & 0 & b\left(K_{\mathrm{DR}}-K_{\mathrm{DL}}\right) \\
0 & m_{\mathrm{w}} & 0 & 0 & 0 \\
0 & 0 & m_{\mathrm{s}}+2 m_{\mathrm{aa}} & 0 & -2 m_{\mathrm{aa}} \cdot h \\
0 & 0 & 0 & m_{\mathrm{w}} & 0 \\
b\left(K_{\mathrm{DR}}-K_{\mathrm{DL}}\right) & 0 & -2 m_{\mathrm{aa}} \cdot h & 0 & \theta_{s x}+2 m_{a a}\left(b^{2}+h^{2}\right)+b^{2}\left(K_{\mathrm{DL}}+K_{\mathrm{DR}}\right) \\
0 & 0 & 0 & 0 & 0 \\
0 & 0 & 0 & 0 & 0 \\
-K_{\mathrm{DL}} & 0 & 0 & 0 & K_{\mathrm{DL}} b \\
-K_{\mathrm{DR}} & 0 & 0 & 0 & -K_{\mathrm{DR}} b \\
0 & 0 & 0 & 0 & 0 \\
0 & 0 & 0 & 0 & 0 \\
0 & 0 & 0 & 0 & 0 \\
0 & 0 & 0 & 0 & 0
\end{array}\right.
$$




$\left.\begin{array}{cccccccc}0 & 0 & 0 & 0 & 0 & 0 & 0 & 0 \\ 0 & 0 & 0 & 0 & 0 & 0 & 0 & 0 \\ 0 & 0 & 0 & 0 & 0 & 0 & 0 & 0 \\ 0 & 0 & 0 & 0 & 0 & 0 & 0 & 0 \\ 0 & 0 & 0 & 0 & 0 & 0 & 0 & 0 \\ 2 m_{\mathrm{v}} & 0 & 0 & 0 & 0 & 0 & 0 & 0 \\ 0 & 2 m_{\mathrm{b}} & 0 & 0 & 0 & 0 & 0 & 0 \\ 0 & 0 & m_{\mathrm{as}}+m_{\mathrm{fL}} & 0 & 0 & 0 & 0 & 0 \\ 0 & 0 & 0 & m_{\mathrm{as}}+m_{\mathrm{fR}} & 0 & 0 & 0 & 0 \\ 0 & 0 & 0 & 0 & 2 m_{\mathrm{v}} & 0 & 0 & 0 \\ 0 & 0 & 0 & 0 & 0 & 2 m_{\mathrm{b}} & 0 & 0 \\ 0 & 0 & 0 & 0 & 0 & 0 & m_{\mathrm{as}}+m_{\mathrm{fL}} & 0 \\ 0 & 0 & 0 & 0 & 0 & 0 & 0 & m_{\mathrm{as}}+m_{\mathrm{fR}}\end{array}\right]$

\section{I-controllers:}

When using I-controllers-with the control parameters $K_{\mathrm{IL}}$ and $K_{\mathrm{IR}}$ for the left side and for the right side of the motor-with negative feedback loops for the vertical motor feet velocities $\dot{z}_{\mathrm{aL}}$ and $\dot{z}_{\mathrm{aR}}$, the actuator forces can described by:

$$
\boldsymbol{f}_{\mathrm{a}}=-\boldsymbol{P}_{\mathrm{azL}} \cdot K_{\mathrm{IL}} \cdot \int \dot{z}_{\mathrm{aL}} \mathrm{d} t-\boldsymbol{P}_{\mathrm{azR}} \cdot K_{\mathrm{IR}} \cdot \int \dot{z}_{\mathrm{aR}} \mathrm{d} t
$$

The differential equation system gets:

$$
\boldsymbol{M} \cdot \ddot{\boldsymbol{q}}+\boldsymbol{D} \cdot \dot{\boldsymbol{q}}+\boldsymbol{C} \cdot \boldsymbol{q}+\boldsymbol{P}_{\mathrm{azL}} \cdot K_{\mathrm{IL}} \cdot\left(z_{\mathrm{s}}-\varphi_{\mathrm{s}} \cdot b\right)+\boldsymbol{P}_{\mathrm{azR}} \cdot K_{\mathrm{IR}} \cdot\left(z_{\mathrm{s}}+\varphi_{\mathrm{s}} \cdot b\right)=\boldsymbol{f}_{\mathrm{e}}
$$

The control parameters $K_{\mathrm{IL}}, K_{\mathrm{IR}}$ can now be integrated into the stiffness matrix, leading to a controlled stiffness matrix $\boldsymbol{C}_{\mathrm{KI}}$. The new differential equation system becomes:

$$
\boldsymbol{M} \cdot \ddot{\boldsymbol{q}}+\boldsymbol{D} \cdot \dot{\boldsymbol{q}}+\boldsymbol{C}_{\mathrm{KI}} \cdot \boldsymbol{q}=\boldsymbol{f}_{\mathrm{e}}
$$

The stiffness matrix $\boldsymbol{C}_{\mathrm{KI}}$ is identical to $\boldsymbol{C}_{\mathrm{KP}}$ regarding the structure, only the controller parameters have to be replaced:

$$
\boldsymbol{C}_{\mathrm{KP}} \stackrel{\substack{K_{\mathrm{PL}} \rightarrow K_{\mathrm{LL}} \\ K_{\mathrm{PR}} \rightarrow K_{\mathrm{RR}}}}{\longrightarrow} \boldsymbol{C}_{\mathrm{KI}}
$$

\section{PI-controllers:}

Using PI-controllers-with the control parameters $K_{\mathrm{PL}}, K_{\mathrm{PR}}$ and $K_{\mathrm{IL}}$, $K_{\mathrm{IR}}$ for the left side and for the right side of the motor-with negative feedback loops for the vertical motor feet velocities $\dot{z}_{\mathrm{aL}}$ and $\dot{z}_{\mathrm{aR}}$, the actuator forces can be described by:

$$
\boldsymbol{f}_{\mathrm{a}}=-\boldsymbol{P}_{\mathrm{azL}} \cdot\left(K_{\mathrm{PL}} \cdot \dot{z}_{\mathrm{aL}}+K_{\mathrm{IL}} \cdot \int \dot{z}_{\mathrm{aL}} \mathrm{d} t\right)-\boldsymbol{P}_{\mathrm{azR}} \cdot\left(K_{\mathrm{PR}} \cdot \dot{z}_{\mathrm{RL}}+K_{\mathrm{IR}} \cdot \int \dot{z}_{\mathrm{aR}} \mathrm{d} t\right)
$$

The differential equation system can be formulated by:

$$
\begin{aligned}
& \boldsymbol{M} \cdot \ddot{\boldsymbol{q}}+\boldsymbol{D} \cdot \dot{\boldsymbol{q}}+\boldsymbol{C} \cdot \boldsymbol{q}+\boldsymbol{P}_{\mathrm{azL}} \cdot\left(K_{\mathrm{PL}} \cdot\left(\dot{z}_{\mathrm{s}}-\dot{\varphi}_{\mathrm{s}} \cdot b\right)+K_{\mathrm{IL}} \cdot\left(z_{\mathrm{s}}-\varphi_{\mathrm{s}} \cdot b\right)\right) \\
& +\boldsymbol{P}_{\mathrm{azR}} \cdot\left(K_{\mathrm{PR}} \cdot\left(\dot{z}_{\mathrm{s}}+\dot{\varphi}_{\mathrm{s}} \cdot b\right)+K_{\mathrm{IR}} \cdot\left(z_{\mathrm{s}}+\varphi_{\mathrm{s}} \cdot b\right)\right)=\boldsymbol{f}_{\mathrm{e}}
\end{aligned}
$$

The control parameters $K_{\mathrm{PL}}, K_{\mathrm{PR}}$ and $K_{\mathrm{IL}}, K_{\mathrm{IR}}$ can now be integrated 
into the stiffness and damping matrix leading to a controlled stiffness matrix $\boldsymbol{C}_{\mathrm{KI}}$ and a controlled damping matrix $\boldsymbol{D}_{\mathrm{KP}}$. The new differential equation system can now be described by:

$$
\boldsymbol{M} \cdot \ddot{\boldsymbol{q}}+\boldsymbol{D}_{\mathrm{KP}} \cdot \dot{\boldsymbol{q}}+\boldsymbol{C}_{\mathrm{KI}} \cdot \boldsymbol{q}=\boldsymbol{f}_{\mathrm{e}}
$$

\section{PID-controllers:}

For ideal PID-controllers-with the control parameters $K_{\mathrm{PL}}, K_{\mathrm{PR}}, K_{\mathrm{IL}}$, $K_{\mathrm{IR}}$ and $K_{\mathrm{DL}}, K_{\mathrm{DR}}$ for the left side and for the right side of the motor-with negative feedback loops for the vertical motor feet velocities $\dot{z}_{\mathrm{aL}}$ and $\dot{z}_{\mathrm{aR}}$, the actuator forces become:

$$
\begin{aligned}
\boldsymbol{f}_{\mathrm{a}}= & -\boldsymbol{P}_{\mathrm{azL}} \cdot\left(K_{\mathrm{PL}} \cdot \dot{z}_{\mathrm{aL}}+K_{\mathrm{IL}} \cdot \int \dot{z}_{\mathrm{aL}} \mathrm{d} t+K_{\mathrm{DL}} \cdot \frac{\mathrm{d} \dot{z}_{\mathrm{aL}}}{\mathrm{d} t}\right) \\
& -\boldsymbol{P}_{\mathrm{azR}} \cdot\left(K_{\mathrm{PR}} \cdot \dot{z}_{\mathrm{RL}}+K_{\mathrm{IR}} \cdot \int \dot{z}_{\mathrm{aR}} \mathrm{d} t+K_{\mathrm{DR}} \cdot \frac{\mathrm{d} \dot{z}_{\mathrm{aR}}}{\mathrm{d} t}\right)
\end{aligned}
$$

Now follows the differential equation system:

$$
\begin{aligned}
& \boldsymbol{M} \cdot \ddot{\boldsymbol{q}}+\boldsymbol{D} \cdot \dot{\boldsymbol{q}}+\boldsymbol{C} \cdot \boldsymbol{q} \\
& +\boldsymbol{P}_{\mathrm{azL}} \cdot\left(K_{\mathrm{PL}} \cdot\left(\dot{z}_{\mathrm{s}}-\dot{\varphi}_{\mathrm{s}} \cdot b\right)+K_{\mathrm{IL}} \cdot\left(z_{\mathrm{s}}-\varphi_{\mathrm{s}} \cdot b\right)+K_{\mathrm{DL}} \cdot\left(\ddot{z}_{\mathrm{s}}-\ddot{\varphi}_{\mathrm{s}} \cdot b\right)\right) \\
& +\boldsymbol{P}_{\mathrm{azR}} \cdot\left(K_{\mathrm{PR}} \cdot\left(\dot{z}_{\mathrm{s}}+\dot{\varphi}_{\mathrm{s}} \cdot b\right)+K_{\mathrm{IR}} \cdot\left(z_{\mathrm{s}}+\varphi_{\mathrm{s}} \cdot b\right)+K_{\mathrm{DR}} \cdot\left(\ddot{z}_{\mathrm{s}}+\ddot{\varphi}_{\mathrm{s}} \cdot b\right)\right)=\boldsymbol{f}_{\mathrm{e}}
\end{aligned}
$$

The control parameters $K_{\mathrm{PL}}, K_{\mathrm{PR}}, K_{\mathrm{IL}}, K_{\mathrm{IR}}$ and $K_{\mathrm{DL}}, K_{\mathrm{DR}}$ can now be integrated into the stiffness, damping and mass matrix leading to a controlled stiffness matrix $\boldsymbol{C}_{\mathrm{KI}}$ and a controlled damping matrix $\boldsymbol{D}_{\mathrm{KP}}$ and a controlled mass matrix $\boldsymbol{M}_{\mathrm{KD}}$. The new differential equation system can now be described by:

$$
\boldsymbol{M}_{\mathrm{KD}} \cdot \ddot{\boldsymbol{q}}+\boldsymbol{D}_{\mathrm{KP}} \cdot \dot{\boldsymbol{q}}+\boldsymbol{C}_{\mathrm{KI}} \cdot \boldsymbol{q}=\boldsymbol{f}_{\mathrm{e}}
$$

\subsubsection{Feedback of the Motor Feet Accelerations}

\section{P-controllers:}

Using P-controllers-with the control parameters $K_{\mathrm{PL}}$ and $K_{\mathrm{PR}}$ for the left side and for the right side of the motor-with negative feedback loops for the vertical motor feet accelerations $\ddot{z}_{\mathrm{aL}}$ and $\ddot{z}_{\mathrm{aR}}$, the actuator forces can described by:

$$
\boldsymbol{f}_{\mathrm{a}}=-\boldsymbol{P}_{\mathrm{azL}} \cdot K_{\mathrm{PL}} \cdot \ddot{z}_{\mathrm{aL}}-\boldsymbol{P}_{\mathrm{azR}} \cdot K_{\mathrm{PR}} \cdot \ddot{z}_{\mathrm{aR}}
$$

The differential equation system can now be described by:

$$
\boldsymbol{M} \cdot \ddot{\boldsymbol{q}}+\boldsymbol{D} \cdot \dot{\boldsymbol{q}}+\boldsymbol{C} \cdot \boldsymbol{q}+\boldsymbol{P}_{\mathrm{azL}} \cdot K_{\mathrm{PL}} \cdot\left(\ddot{z}_{\mathrm{s}}-\ddot{\varphi}_{\mathrm{s}} \cdot b\right)+\boldsymbol{P}_{\mathrm{azR}} \cdot K_{\mathrm{PR}} \cdot\left(\ddot{z}_{\mathrm{s}}+\ddot{\varphi}_{\mathrm{s}} \cdot b\right)=\boldsymbol{f}_{\mathrm{e}}
$$

The control parameters $K_{\mathrm{PL}}, K_{\mathrm{PR}}$ can now be integrated into the mass matrix, leading to a controlled mass matrix $\boldsymbol{M}_{\mathrm{KP}}$. The new differential equation system becomes:

$$
\boldsymbol{M}_{\mathrm{KP}} \cdot \ddot{\boldsymbol{q}}+\boldsymbol{D} \cdot \dot{\boldsymbol{q}}+\boldsymbol{C} \cdot \boldsymbol{q}=\boldsymbol{f}_{\mathrm{e}}
$$

The mass matrix $\boldsymbol{M}_{\mathrm{KP}}$ is identical to $\boldsymbol{M}_{\mathrm{KD}}$ regarding the structure, only the controller parameters have to be replaced: 


$$
\boldsymbol{M}_{\mathrm{KD}} \stackrel{\substack{K_{\mathrm{DL}} \rightarrow K_{\mathrm{PL}} \\ K_{\mathrm{DR}} \rightarrow K_{\mathrm{PR}}}}{\longrightarrow} \boldsymbol{M}_{\mathrm{KP}}
$$

\section{I-controllers:}

For I-controllers-with the control parameters $K_{\mathrm{IL}}$ and $K_{\mathrm{IR}}$ for the left side and for the right side of the motor-with negative feedback loops for the accelerations $\ddot{z}_{\mathrm{aL}}$ and $\ddot{z}_{\mathrm{aR}}$, the actuator forces can described by:

$$
\boldsymbol{f}_{\mathrm{a}}=-\boldsymbol{P}_{\mathrm{azL}} \cdot K_{\mathrm{IL}} \cdot \int \ddot{z}_{\mathrm{aL}} \mathrm{d} t-\boldsymbol{P}_{\mathrm{azR}} \cdot K_{\mathrm{IR}} \cdot \int \ddot{z}_{\mathrm{aR}} \mathrm{d} t
$$

Now follows the differential equation system:

$$
\boldsymbol{M} \cdot \ddot{\boldsymbol{q}}+\boldsymbol{D} \cdot \dot{\boldsymbol{q}}+\boldsymbol{C} \cdot \boldsymbol{q}+\boldsymbol{P}_{\mathrm{azL}} \cdot K_{\mathrm{IL}} \cdot\left(\dot{z}_{\mathrm{s}}-\dot{\varphi}_{\mathrm{s}} \cdot b\right)+\boldsymbol{P}_{\mathrm{azR}} \cdot K_{\mathrm{IR}} \cdot\left(\dot{z}_{\mathrm{s}}+\dot{\varphi}_{\mathrm{s}} \cdot b\right)=\boldsymbol{f}_{\mathrm{e}}
$$

The control parameters $K_{\mathrm{IL}}, K_{\mathrm{IR}}$ can now be integrated into the damping matrix leading to a controlled damping matrix $\boldsymbol{D}_{\mathrm{KI}}$. The new differential equation system can now be described by:

$$
\boldsymbol{M} \cdot \ddot{\boldsymbol{q}}+\boldsymbol{D}_{\mathrm{KI}} \cdot \dot{\boldsymbol{q}}+\boldsymbol{C} \cdot \boldsymbol{q}=\boldsymbol{f}_{\mathrm{e}}
$$

The stiffness matrix $\boldsymbol{D}_{\mathrm{KI}}$ is identical to $\boldsymbol{D}_{\mathrm{KD}}$ regarding the structure, only the controller parameters have to be replaced:

$$
\boldsymbol{D}_{\mathrm{KD}} \stackrel{\substack{K_{\mathrm{DL}} \rightarrow K_{\mathrm{IL}} \\ K_{\mathrm{DR}} \rightarrow K_{\mathrm{IR}}}}{\longrightarrow} \boldsymbol{D}_{\mathrm{KI}}
$$

\section{PI-controllers:}

Using PI-controllers-with the control parameters $K_{\mathrm{PL}}, K_{\mathrm{PR}}$ and $K_{\mathrm{IL}}$, $K_{\mathrm{IR}}$ for the left side and for the right side of the motor-with negative feedback loops for the vertical motor feet accelerations $\ddot{z}_{\mathrm{aL}}$ and $\ddot{z}_{\mathrm{aR}}$, the actuator forces become:

$$
\boldsymbol{f}_{\mathrm{a}}=-\boldsymbol{P}_{\mathrm{azL}} \cdot\left(K_{\mathrm{PL}} \cdot \ddot{z}_{\mathrm{aL}}+K_{\mathrm{IL}} \cdot \int \ddot{z}_{\mathrm{aL}} \mathrm{d} t\right)-\boldsymbol{P}_{\mathrm{azR}} \cdot\left(K_{\mathrm{PR}} \cdot \ddot{z}_{\mathrm{RL}}+K_{\mathrm{IR}} \cdot \int \ddot{z}_{\mathrm{aR}} \mathrm{d} t\right)
$$

The differential equation system can be written by:

$$
\begin{aligned}
& \boldsymbol{M} \cdot \ddot{\boldsymbol{q}}+\boldsymbol{D} \cdot \dot{\boldsymbol{q}}+\boldsymbol{C} \cdot \boldsymbol{q}+\boldsymbol{P}_{\mathrm{azL}} \cdot\left(K_{\mathrm{PL}} \cdot\left(\ddot{z}_{\mathrm{s}}-\ddot{\varphi}_{\mathrm{s}} \cdot b\right)+K_{\mathrm{IL}} \cdot\left(\dot{z}_{\mathrm{s}}-\dot{\varphi}_{\mathrm{s}} \cdot b\right)\right) \\
& +\boldsymbol{P}_{\mathrm{azR}} \cdot\left(K_{\mathrm{PR}} \cdot\left(\ddot{z}_{\mathrm{s}}+\ddot{\varphi}_{\mathrm{s}} \cdot b\right)+K_{\mathrm{IR}} \cdot\left(\dot{z}_{\mathrm{s}}+\dot{\varphi}_{\mathrm{s}} \cdot b\right)\right)=\boldsymbol{f}_{\mathrm{e}}
\end{aligned}
$$

The control parameters $K_{\mathrm{PL}}, K_{\mathrm{PR}}$ and $K_{\mathrm{IL}}, K_{\mathrm{IR}}$ can now be integrated into the mass and damping matrix leading to a controlled mass matrix $\boldsymbol{M}_{\mathrm{KP}}$ and a controlled damping matrix $\boldsymbol{D}_{\mathrm{KI}}$.

The new differential equation system can now be described by:

$$
\boldsymbol{M}_{\mathrm{KP}} \cdot \ddot{\boldsymbol{q}}+\boldsymbol{D}_{\mathrm{KI}} \cdot \dot{\boldsymbol{q}}+\boldsymbol{C} \cdot \boldsymbol{q}=\boldsymbol{f}_{\mathrm{e}}
$$

\subsubsection{Summery Regarding the Control Strategies}

In summary, the vibration system can be described by the following differential equation:

$$
\tilde{\boldsymbol{M}} \cdot \ddot{\boldsymbol{q}}+\tilde{\boldsymbol{D}} \cdot \dot{\boldsymbol{q}}+\tilde{\boldsymbol{C}} \cdot \boldsymbol{q}=\boldsymbol{f}_{\mathrm{e}}
$$

The corresponding matrices, depending on the chosen controller structure and the chosen feedback, are shown in Table 1.

The cells marked with "_-" cannot be performed, if the above described structure of the differential equation system (59) shall be used. Some 
Table 1. Matrices, depending on controller structure and feedback.

\begin{tabular}{|c|c|c|c|c|}
\hline Controllers & Displacement feedback & Velocity feedback & Acceleration feedback & Open Loop \\
\hline \multirow{3}{*}{$\mathrm{P}$} & $\tilde{\boldsymbol{M}}=\boldsymbol{M}$ & $\tilde{\boldsymbol{M}}=\boldsymbol{M}$ & $\tilde{\boldsymbol{M}}=\boldsymbol{M}_{\mathrm{KP}}$ & \\
\hline & $\tilde{D}=\boldsymbol{D}$ & $\tilde{\boldsymbol{D}}=\boldsymbol{D}_{\mathrm{KP}}$ & $\tilde{D}=D$ & \\
\hline & $\tilde{\boldsymbol{C}}=\boldsymbol{C}_{\mathrm{KP}}$ & $\tilde{\boldsymbol{C}}=\boldsymbol{C}$ & $\tilde{\boldsymbol{C}}=\boldsymbol{C}$ & \\
\hline \multirow{3}{*}{ PD (ideal) } & $\tilde{\boldsymbol{M}}=\boldsymbol{M}$ & $\tilde{\boldsymbol{M}}=\boldsymbol{M}_{\mathrm{KD}}$ & & \\
\hline & $\tilde{\boldsymbol{D}}=\boldsymbol{D}_{\mathrm{KD}}$ & $\tilde{\boldsymbol{D}}=\boldsymbol{D}_{\mathrm{KP}}$ & - & \\
\hline & $\tilde{\boldsymbol{C}}=\boldsymbol{C}_{\mathrm{KP}}$ & $\tilde{\boldsymbol{C}}=\boldsymbol{C}$ & & \\
\hline \multirow{3}{*}{ I } & \multirow{3}{*}{ - } & $\tilde{\boldsymbol{M}}=\boldsymbol{M}$ & $\tilde{\boldsymbol{M}}=\boldsymbol{M}$ & $\tilde{\boldsymbol{M}}=\boldsymbol{M}$ \\
\hline & & $\tilde{\boldsymbol{D}}=\boldsymbol{D}$ & $\tilde{\boldsymbol{D}}=\boldsymbol{D}_{\mathrm{KI}}$ & $\tilde{\boldsymbol{D}}=\boldsymbol{D}$ \\
\hline & & $\tilde{\boldsymbol{C}}=\boldsymbol{C}_{\mathrm{KI}}$ & $\tilde{\boldsymbol{C}}=\boldsymbol{C}$ & $\tilde{\boldsymbol{C}}=\boldsymbol{C}$ \\
\hline PI & - & $\begin{aligned} \tilde{\boldsymbol{M}} & =\boldsymbol{M} \\
\tilde{\boldsymbol{D}} & =\boldsymbol{D}_{\mathrm{KP}} \\
\tilde{\boldsymbol{C}} & =\boldsymbol{C}_{\mathrm{KI}}\end{aligned}$ & $\begin{aligned} \tilde{\boldsymbol{M}} & =\boldsymbol{M}_{\mathrm{KP}} \\
\tilde{\boldsymbol{D}} & =\boldsymbol{D}_{\mathrm{KI}} \\
\tilde{\boldsymbol{C}} & =\boldsymbol{C}\end{aligned}$ & \\
\hline PID (ideal) & - & $\begin{aligned} \tilde{\boldsymbol{M}} & =\boldsymbol{M}_{\mathrm{KD}} \\
\tilde{\boldsymbol{D}} & =\boldsymbol{D}_{\mathrm{KP}} \\
\tilde{\boldsymbol{C}} & =\boldsymbol{C}_{\mathrm{KI}}\end{aligned}$ & - & \\
\hline
\end{tabular}

combinations lead to the same differential equation, if the control parameters are identically.

For example: P-controllers with displacement feedback lead to the same results as I-controllers with velocity feedback, if the following boundary condition is given for the control parameters:

$$
K_{\mathrm{PL}}=K_{\mathrm{IL}} ; K_{\mathrm{PR}}=K_{\mathrm{IR}}
$$

\subsection{Stability Analysis}

A stability analysis has to be performed, because there are different kinds of sources of instability, as the oil film of the sleeve bearings, the rotating damping of the rotor shaft, the electromagnetic field damping, and the control system. To derive the eigenvalues, the homogenous differential equation has to be solved:

$$
\tilde{\boldsymbol{M}} \cdot \ddot{\boldsymbol{q}}+\tilde{\boldsymbol{D}} \cdot \dot{\boldsymbol{q}}+\tilde{\boldsymbol{C}} \cdot \boldsymbol{q}=\mathbf{0}
$$

Therefore, the differential equation can be transferred into the state space formulation, according to [7]:

$$
\begin{aligned}
& {\left[\begin{array}{c}
\dot{\boldsymbol{q}} \\
\ddot{\boldsymbol{q}}
\end{array}\right]=\left[\begin{array}{cc}
\mathbf{0} & \boldsymbol{I} \\
-\tilde{\boldsymbol{M}}^{-1} \cdot \tilde{\boldsymbol{C}} & -\tilde{\boldsymbol{M}}^{-1} \cdot \tilde{\boldsymbol{D}}
\end{array}\right] \cdot \underbrace{\left[\begin{array}{c}
\boldsymbol{q} \\
\dot{\boldsymbol{q}}
\end{array}\right]}_{\boldsymbol{x}}} \\
& \text { With the ansatz } \boldsymbol{x}=\hat{\boldsymbol{x}} \cdot e^{\lambda \cdot t}
\end{aligned}
$$

the eigenvalues $\lambda$ can be calculated by:

$$
\operatorname{det}\left[\left[\begin{array}{cc}
\mathbf{0} & \boldsymbol{I} \\
-\tilde{\boldsymbol{M}}^{-1} \cdot \tilde{\boldsymbol{C}} & -\tilde{\boldsymbol{M}}^{-1} \cdot \tilde{\boldsymbol{D}}
\end{array}\right]-\lambda \cdot \boldsymbol{I}\right]=0
$$

When the eigenvalues have been derived, the real parts of all eigenvalues have to be analyzed. If all real parts are negative, the system is stable. It has to be consi- 
dered here, that for calculating the damping values-using the equations with the loss factors-the whirling frequency $\omega_{\mathrm{F}}$ has to be defined. An iterative algorithm [20] can be executed, where the whirling frequency of the mode, which gets instable, will be used for deriving the frequency dependent parameters.

\subsection{Forced Vibration Analysis}

To derive the complex amplitude vectors of the coordinate vector for each kind of excitation, following equation has to be solved:

$$
\hat{\boldsymbol{q}}_{\mathrm{k}}=\left[-\tilde{\boldsymbol{M}} \cdot \Omega^{2}+\tilde{\boldsymbol{D}} \cdot j \cdot \Omega+\tilde{\boldsymbol{C}}\right]^{-1} \cdot \hat{\boldsymbol{f}}_{\mathrm{e}, \mathrm{\kappa}}
$$

The index $\kappa$ is used to describe the different kinds of excitation, for mass eccentricity $\kappa=u$, for bent rotor deflection $\kappa=a$ and for magnetic eccentricity $\kappa=m$.

The solution regarding the coordinate vector for each excitation can now be described by:

$$
\boldsymbol{q}_{\mathrm{K}}=\hat{\boldsymbol{q}}_{\mathrm{K}} \cdot e^{j\left(\Omega \cdot t+\varphi_{\mathrm{K}}\right)}
$$

with:

$$
\hat{\boldsymbol{q}}_{\mathrm{\kappa}}=\left[\hat{z}_{\mathrm{s}, \kappa} ; \hat{z}_{\mathrm{w}, \kappa} ; \hat{y}_{\mathrm{s}, \kappa} ; \hat{y}_{\mathrm{w}, \kappa} ; \hat{\varphi}_{\mathrm{s}, \kappa} ; \hat{z}_{\mathrm{v}, \mathrm{\kappa}} ; \hat{z}_{\mathrm{b}, \mathrm{\kappa}} ; \hat{z}_{\mathrm{fL}, \kappa} ; \hat{\mathrm{fR}}_{\mathrm{fR}, \mathrm{\kappa}} ; \hat{y}_{\mathrm{v}, \mathrm{\kappa}} ; \hat{y}_{\mathrm{b}, \kappa} ; \hat{y}_{\mathrm{fL}, \kappa} ; \hat{y}_{\mathrm{fR}, \mathrm{\kappa}}\right]^{\mathrm{T}}
$$

Now the bearing housing vibration velocities can be derived for vertical $(z)$ and horizontal $(y)$ direction, for each kind of excitation:

- Vertical: $v_{\mathrm{b}, \mathrm{z}, \mathrm{\kappa}}=\Omega \cdot\left|\hat{z}_{\mathrm{b}, \mathrm{\kappa}}\right|$ (68)

- Horizontal: $v_{\mathrm{b}, \mathrm{y}, \mathrm{\kappa}}=\Omega \cdot\left|\hat{y}_{\mathrm{b}, \mathrm{\kappa}}\right|(69)$

In the same way, the foundation vibration velocities for each motor side in vertical and horizontal direction, for each kind of excitation, can be calculated:

- Left side, vertical: $v_{\mathrm{fL}, \mathrm{z}, \mathrm{K}}=\Omega \cdot\left|\hat{\mathrm{z}}_{\mathrm{fL}, \mathrm{\kappa}}\right| \quad(70)$

- Left side, horizontal: $v_{\mathrm{fL}, \mathrm{y}, \mathrm{\kappa}}=\Omega \cdot\left|\hat{y}_{\mathrm{fL}, \mathrm{\kappa}}\right| \quad(71)$

- Right side, vertical: $v_{\mathrm{fR}, z, \mathrm{k}}=\Omega \cdot\left|\hat{z}_{\mathrm{fR}, \mathrm{\kappa}}\right|$ (72)

- Right side, horizontal: $v_{\mathrm{fR}, \mathrm{y}, \mathrm{\kappa}}=\Omega \cdot\left|\hat{y}_{\mathrm{fR}, \mathrm{\kappa}}\right|$ (73)

The actuator forces depend on the control strategy and the feedback loop. E.g. for PID-controllers with a velocity feedback, the active actuator forces for each motor side can be calculated by:

- Left side: $f_{\mathrm{azL}, \mathrm{\kappa}}=\hat{f}_{\mathrm{azL}, \mathrm{\kappa}} \cdot e^{j \Omega t} \quad(74)$ with:

$$
\hat{f}_{\mathrm{azL}, \mathrm{\kappa}}=-\left(-K_{\mathrm{DL}} \cdot \Omega^{2}+K_{\mathrm{PL}} \cdot j \Omega+K_{\mathrm{IL}}\right) \cdot\left(\hat{z}_{\mathrm{s}, \mathrm{\kappa}}-\hat{\varphi}_{\mathrm{s}, \mathrm{\kappa}} \cdot b\right)
$$

- Right side: $f_{\mathrm{azR}, \mathrm{\kappa}}=\hat{f}_{\mathrm{azR}, \mathrm{\kappa}} \cdot e^{j \Omega t} \quad$ (76)

with:

$$
\hat{f}_{\mathrm{azR}, \mathrm{\kappa}}=-\left(-K_{\mathrm{DR}} \cdot \Omega^{2}+K_{\mathrm{PR}} \cdot j \Omega+K_{\mathrm{IR}}\right) \cdot\left(\hat{z}_{\mathrm{s}, \mathrm{\kappa}}+\hat{\varphi}_{\mathrm{s}, \mathrm{\kappa}} \cdot b\right)
$$

\section{Numerical Example}

A numerical example is now presented, where the vibration velocities of the bearing housings and of the foundation are analyzed as well as the actuator forces, for different control strategies. 


\subsection{Boundary Conditions}

The induction motor is 2-pole motor, converter driven, with sleeve bearings and a stiff rotor design. The load machine has a quadratic function of the load torque $M_{\mathrm{L}}(n)$, in respect to the rotor speed $n$. The induction motor operates between $300 \mathrm{rpm}$ and $3600 \mathrm{rpm}$, where the motor is driven with constant magnetization. Above $3600 \mathrm{rpm}$, up to $4500 \mathrm{rpm}$, the motor operates in the field weakening range, which belongs not to the operating speed range. The data are shown in Table 2.

Table 2. Main data of the 2-pole induction motor, foundation and actuators.

\begin{tabular}{|c|c|}
\hline Data of the motor: & \\
\hline Rated power & $P_{\mathrm{N}}=2400 \mathrm{~kW}$ \\
\hline Rated speed & $n_{\mathrm{N}}=3600 \mathrm{rpm}$ \\
\hline Masse of the stator & $m_{\mathrm{s}}=7040 \mathrm{~kg}$ \\
\hline Mass inertia of the stator at the $x$-axis & $\theta_{\mathrm{sx}}=1550 \mathrm{~kg} \cdot \mathrm{m}^{2}$ \\
\hline Mass of the rotor & $m_{\mathrm{w}}=1900 \mathrm{~kg}$ \\
\hline Mass of the rotor shaft journal & $m_{\mathrm{v}}=10 \mathrm{~kg}$ \\
\hline Mass of the bearing housing & $m_{\mathrm{b}}=80 \mathrm{~kg}$ \\
\hline Stiffness of the rotor & $c=6.0 \times 10^{8} \mathrm{~kg} / \mathrm{s}^{2}$ \\
\hline Height of the centre of gravity $S$ & $h=560 \mathrm{~mm}$ \\
\hline Distance between motor feet & $2 b=1060 \mathrm{~mm}$ \\
\hline Horizontal stiffness of bearing housing and end shield & $c_{\text {by }}=4.8 \times 10^{8} \mathrm{~kg} / \mathrm{s}^{2}$ \\
\hline Vertical stiffness of bearing housing and end shield & $c_{\mathrm{bz}}=5.7 \times 10^{8} \mathrm{~kg} / \mathrm{s}^{2}$ \\
\hline Mechanical loss factor of the bearing housing and end shield & $\tan \delta_{\mathrm{b}}=0.04$ \\
\hline Mechanical loss factor of the rotor & $\tan \delta_{\mathrm{i}}=0.03$ \\
\hline Data of the sleeve bearings: & \\
\hline Bearing shell & Cylindrical \\
\hline Lubricant viscosity grade & ISO VG 32 \\
\hline Nominal bore diameter/Bearing width & $d_{\mathrm{b}}=110 \mathrm{~mm} / b_{\mathrm{b}}=81.4 \mathrm{~mm}$ \\
\hline Ambient temperature/Supply oil temperature & $T_{\mathrm{amb}}=20^{\circ} \mathrm{C} / T_{\mathrm{in}}=40^{\circ} \mathrm{C}$ \\
\hline Mean relative bearing clearance (DIN 31698) & $\Psi_{\mathrm{m}}=1.6 \%$ \\
\hline Data of the foundation (for each motor side): & \\
\hline Mass left side & $m_{\mathrm{fL}}=30 \mathrm{~kg}$ \\
\hline Mass right side & $m_{\mathrm{fR}}=30 \mathrm{~kg}$ \\
\hline Vertical stiffness for each motor side & $c_{\mathrm{fzL}}=c_{\mathrm{fzR}}=1.5 \times 10^{8} \mathrm{~kg} / \mathrm{s}^{2}$ \\
\hline Horizontal stiffness for each motor side & $c_{\mathrm{fyL}}=c_{\mathrm{fyR}}=1.0 \times 10^{8} \mathrm{~kg} / \mathrm{s}^{2}$ \\
\hline Mechanical loss factor & $\tan \delta_{\mathrm{f}}=0.04$ \\
\hline Data of the actuators (for each motor side): & \\
\hline Mass of the stator & $m_{\mathrm{as}}=10 \mathrm{~kg}$ \\
\hline Mass of the armature & $m_{\mathrm{aa}}=3 \mathrm{~kg}$ \\
\hline Vertical stiffness & $c_{\mathrm{az}}=1.2 \times 10^{8} \mathrm{~kg} / \mathrm{s}^{2}$ \\
\hline Horizontal stiffness & $c_{\text {ay }}=3.0 \times 10^{8} \mathrm{~kg} / \mathrm{s}^{2}$ \\
\hline Mechanical loss factor & $\tan \delta_{a}=0.04$ \\
\hline
\end{tabular}


The oil film stiffness and damping coefficients of the sleeve bearings are pictured in Figure 3.

The damped electromagnetic spring coefficient and the electromagnetic damper coefficient for forced vibration due to dynamic eccentricity are pictured in Figure 4, where the field weakening operation range, which starts at $3600 \mathrm{rpm}$, is clearly obvious.

The excitation which is here investigated is the bent rotor deflection, so that the differential equation becomes here:

$$
\tilde{\boldsymbol{M}} \cdot \ddot{\boldsymbol{q}}+\tilde{\boldsymbol{D}} \cdot \dot{\boldsymbol{q}}+\tilde{\boldsymbol{C}} \cdot \boldsymbol{q}=\boldsymbol{f}_{\mathrm{e}, \mathrm{a}}
$$

For the forced vibration analysis the bearing housing vibration velocities and the foundation vibration velocities as well as the actuator forces-all related on the bent rotor deflection a-are calculated here in the speed range from $300 \mathrm{rpm}$ up to $4500 \mathrm{rpm}$ for different control strategies. Instability was also investigated, so that it can be pointed out that in den considered ranges the system is stable, but is not pictured here, because it is not the focus of the paper. For investigation of the influence of the control system, P-controllers in conjunction with feedback loops for the motor feet displacements, motor feet velocities and motor feet accelerations are used. Therefore, the influence of each separate controlled matrix-controlled stiffness matrix $\boldsymbol{C}_{\mathrm{KP}}$, controlled mass matrix $\boldsymbol{M}_{\mathrm{KP}}$ and controlled damping matrix $\boldsymbol{D}_{\mathrm{KP}}$-can be analyzed.

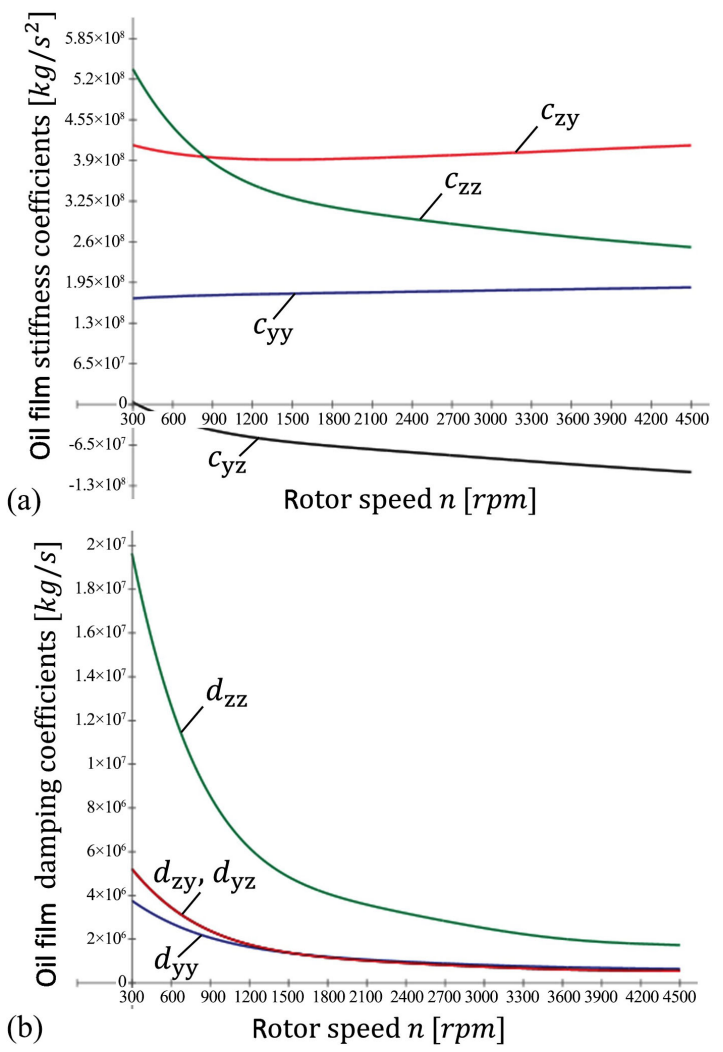

Figure 3. Oil film stiffness (a) and damping coefficients

(b) of the sleeve bearings, depending on the rotor speed. 


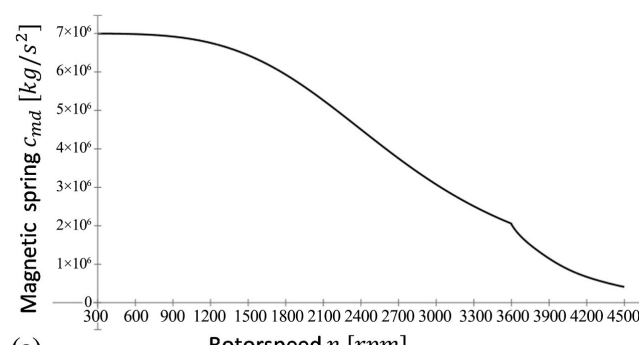

(a)

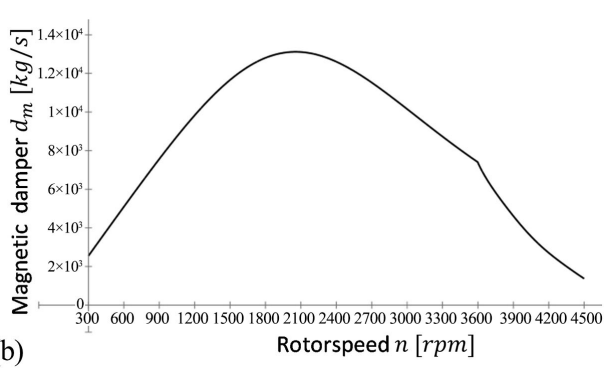

(b)

Figure 4. Damped electromagnetic spring coefficient (a) and the electromagnetic damper coefficient (b) for forced vibration $\left(\omega_{\mathrm{F}}=\Omega\right.$ ) due to dynamic eccentricity, depending on the rotor speed.

\subsection{P-Controllers with Displacement Feedback}

In this case the differential equation system becomes:

$$
\boldsymbol{M} \cdot \ddot{\boldsymbol{q}}+\boldsymbol{D} \cdot \dot{\boldsymbol{q}}+\boldsymbol{C}_{\mathrm{KP}} \cdot \boldsymbol{q}=\boldsymbol{f}_{\mathrm{e}, \mathrm{a}}
$$

The control parameters are here considered in the range:

$$
K_{\mathrm{p}}=K_{\mathrm{pL}}=K_{\mathrm{pR}}=0 \cdots 10^{9} \mathrm{~kg} / \mathrm{s}^{2}
$$

The control parameters and are here integrated into the controlled stiffness matrix $\boldsymbol{C}_{\mathrm{KP}}$. The related bearing housing vibration velocities are pictured in Figure 5.

Figure 5 shows, that, with increasing the control parameter $K_{\mathrm{p}}$, the resonances regarding the bearing housing vibrations can be shifted to higher speeds. For the vertical bearing housing vibration, the resonances can be shifted from about $1140 \mathrm{rpm}$ to $3200 \mathrm{rpm}$. The resonances for the horizontal bearing housing vibration can be shifted from about $745 \mathrm{rpm}$ to $1125 \mathrm{rpm}$ and from about 2390 rpm to $4500 \mathrm{rpm}$. However, the amplitudes in the resonances are also changing, mostly to higher values. The related foundation vibration velocities are pictured in Figure 6. Here also the resonances are also clearly shifted to higher rotor speeds by increasing the control parameter $K_{\mathrm{p}}$. The amplitudes in the resonances get very high, by shifting the resonances to higher speeds.

In Figure 7, the absolute values of the actuator forces are pictured, leading to high actuator forces in the resonances of the vertical foundation vibrations.

\subsection{P-Controllers with Acceleration Feedback}

Now, the differential equation system becomes:

$$
\boldsymbol{M}_{\mathrm{KP}} \cdot \ddot{\boldsymbol{q}}+\boldsymbol{D} \cdot \dot{\boldsymbol{q}}+\boldsymbol{C} \cdot \boldsymbol{q}=\boldsymbol{f}_{\mathrm{e}, \mathrm{a}}
$$

The control parameters are here considered in the range:

$$
K_{\mathrm{p}}=K_{\mathrm{pL}}=K_{\mathrm{pR}}=0 \cdots 10^{4} \mathrm{~kg}
$$

The control parameters are here integrated into the controlled mass matrix $\boldsymbol{M}_{\mathrm{KP}}$. The related bearing housing vibration velocities are pictured in Figure 8 .

Figure 8 shows, that, with increasing the control parameter $K_{\mathrm{p}}$, the resonances regarding the bearing housing vibrations are shifted to lower speeds. For 
the vertical bearing housing vibration, the resonance is shifted from about 1140 rpm to $772 \mathrm{rpm}$. The resonances for the horizontal bearing housing vibrations are shifted from about $745 \mathrm{rpm}$ to $628 \mathrm{rpm}$ and from about $2390 \mathrm{rpm}$ to 1630 rpm.
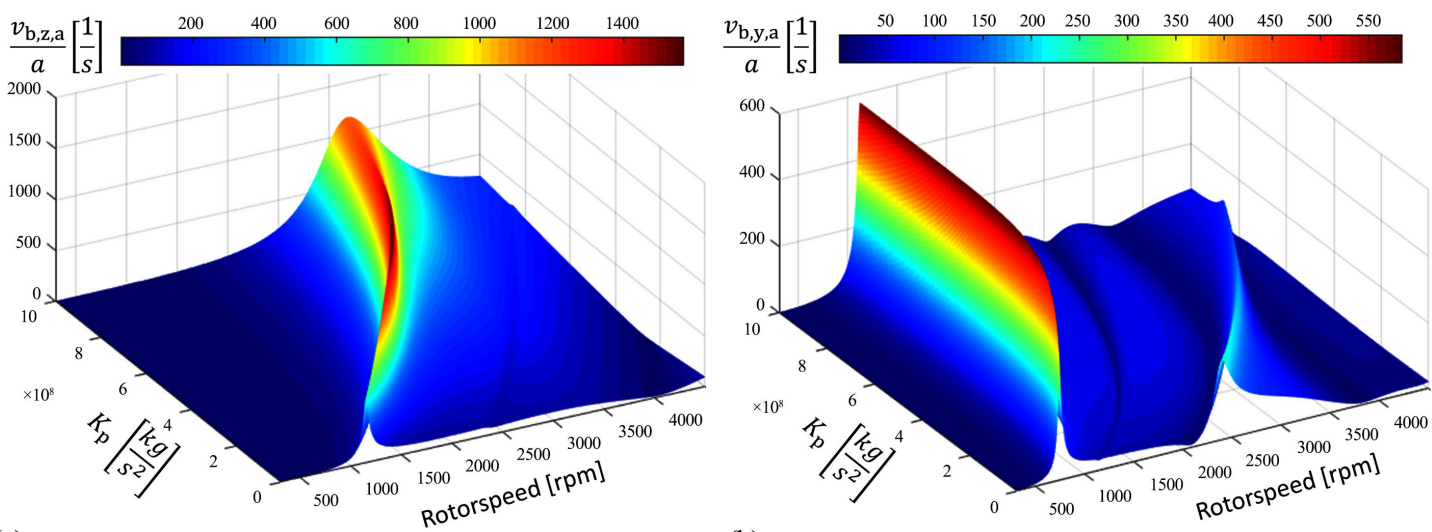

(a)

(b)

Figure 5. Vertical (a) and horizontal (b) bearing housing vibration velocities, related on bent rotor deflection, for different rotor speeds and control parameters, for P-controllers with displacement feedback.
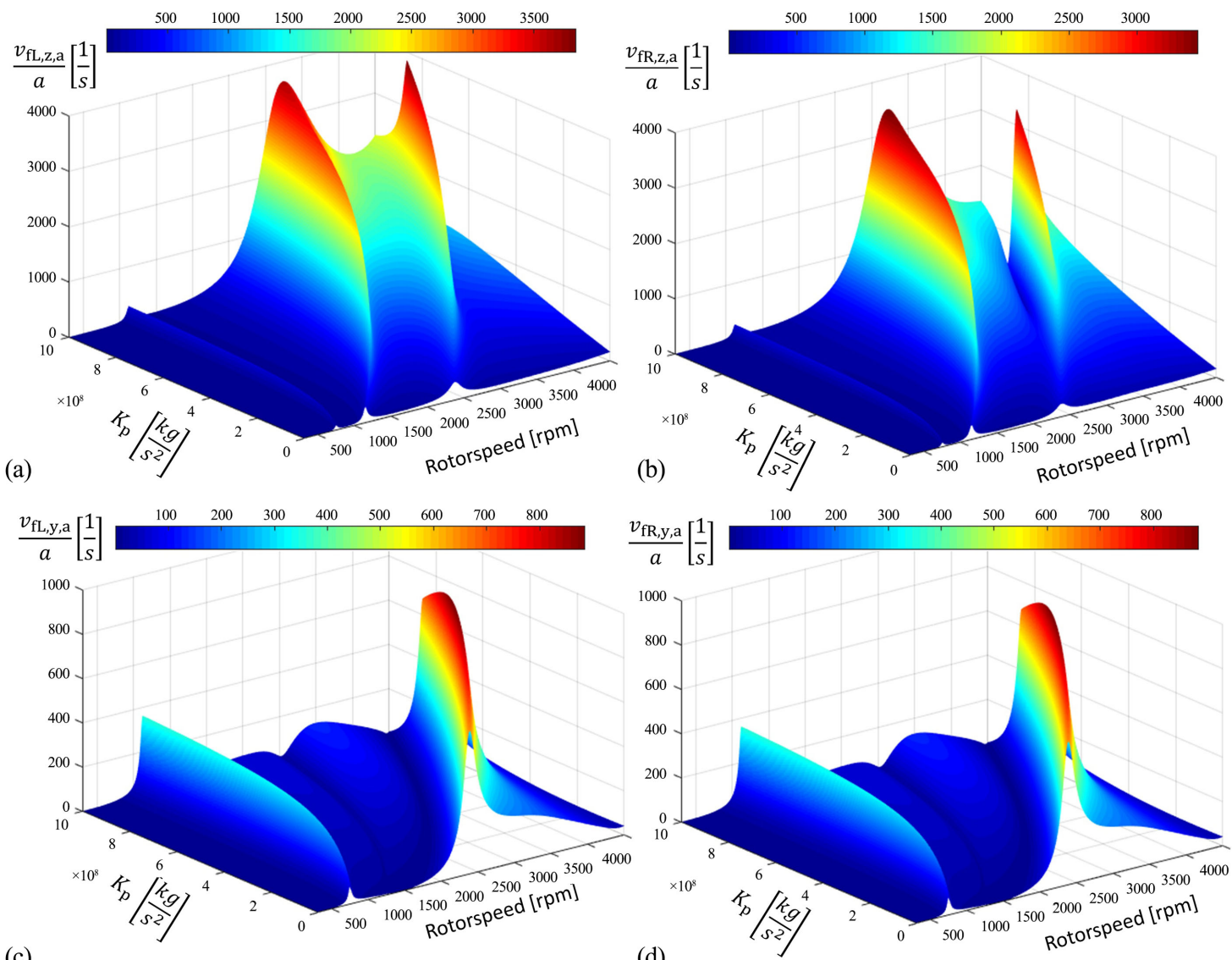

(c)

(d)

Figure 6. Vertical left side (a) and vertical right side (b) foundation vibration velocities and horizontal left side (c) and horizontal right side (d) foundation vibration velocities, related on bent rotor deflection, for different rotor speeds and control parameters, for P-controllers with displacement feedback. 

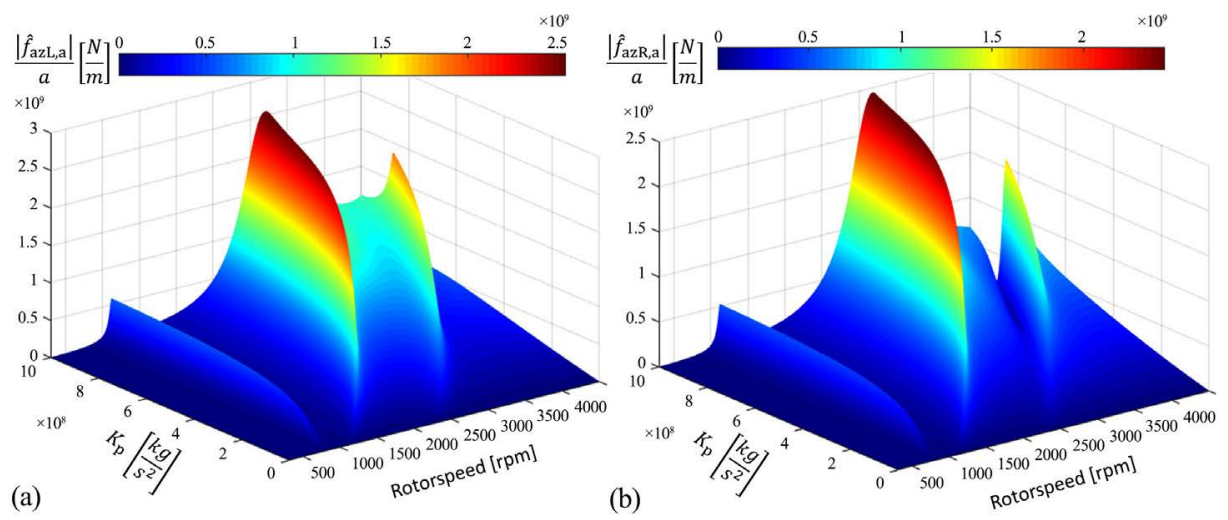

Figure 7. Absolute values of the vertical actuator forces (a) left side and (b) right side, related on bent rotor deflection, for different rotor speeds and control parameters, for P-controllers with displacement feedback.

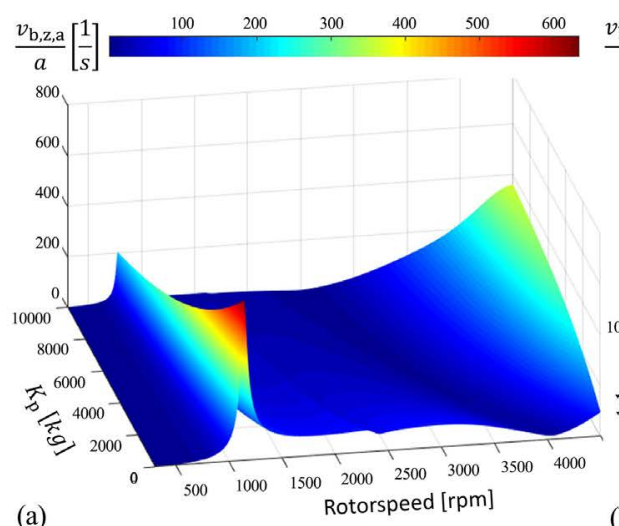

(a)

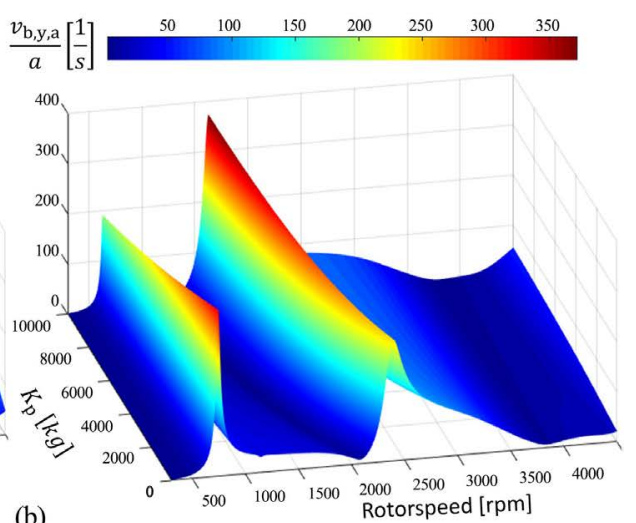

(b)

Figure 8. Vertical (a) and horizontal (b) bearing housing vibration velocities, related on bent rotor deflection, for different rotor speeds and control parameters, for P-controllers with acceleration feedback.

The related foundation vibration velocities are pictured in Figure 9. Here also the resonances are shifted to lower rotor speeds with increasing the control parameter $K_{\mathrm{p}}$. Regarding the vertical foundation vibrations-Figure 9(a) and Figure $9(\mathrm{~b})$ - very high foundation vibrations occur for high rotor speeds in combination with large values of $K_{\mathrm{p}}$. For the horizontal foundation vibrations-Figure 9(c) and Figure 9(d)-the dominating critical speed at about $2390 \mathrm{rpm}$ can be shifted to $1630 \mathrm{rpm}$.

The absolute values of the actuator forces are pictured in Figure 10, leading to high actuator forces for high rotor speeds in combination with large values of $K_{\mathrm{p}}$, corresponding to the high vertical foundation vibrations in Figure 9(a) and Figure 9(b).

\subsection{P-Controllers with Velocity Feedback}

Now, the differential equation system becomes:

$$
\boldsymbol{M} \cdot \ddot{\boldsymbol{q}}+\boldsymbol{D}_{\mathrm{KP}} \cdot \dot{\boldsymbol{q}}+\boldsymbol{C} \cdot \boldsymbol{q}=\boldsymbol{f}_{\mathrm{e}, \mathrm{a}}
$$

The control parameters are here considered in the range: 

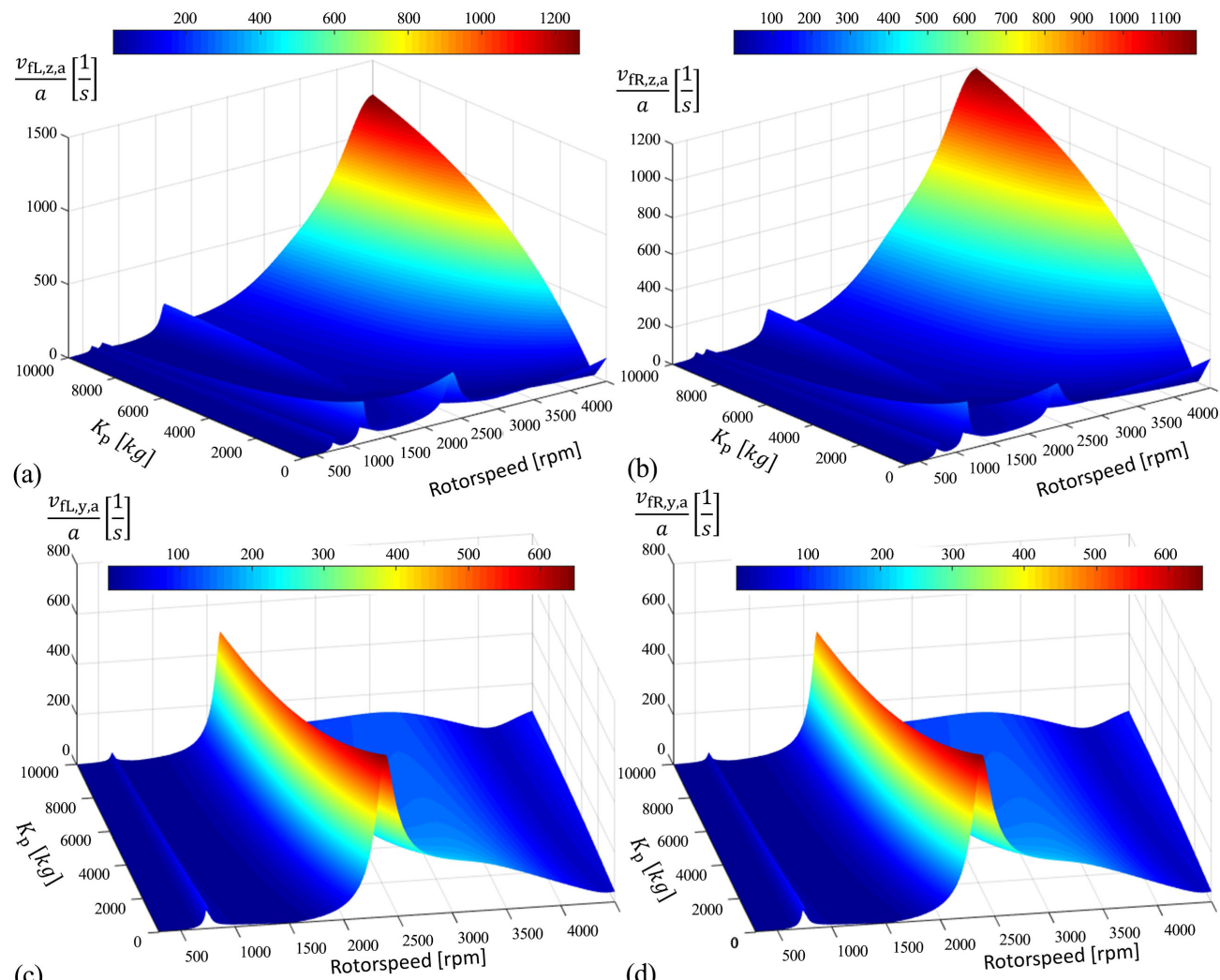

(b)

$\frac{v_{\mathrm{fR}, \mathrm{y}, \mathrm{a}}}{a}\left[\frac{1}{s}\right]$

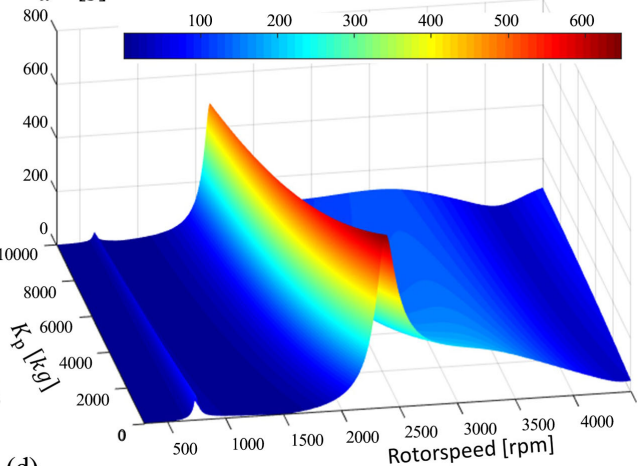

(d)

Figure 9. Vertical left side (a) and vertical right side (b) foundation vibration velocities and horizontal left side (c) and horizontal right side (d) foundation vibration velocities, related on bent rotor deflection, for different rotor speeds and control parameters, for P-controllers with acceleration feedback.
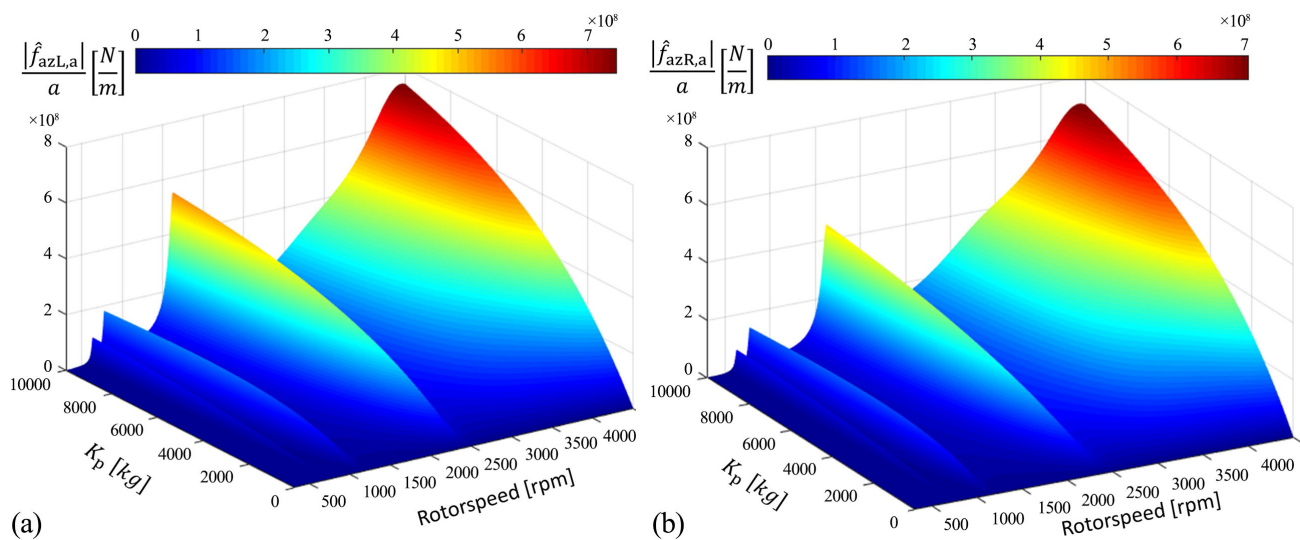

Figure 10. Absolute values of the vertical actuator forces (a) left side and (b) right side, related on bent rotor deflection, for different rotor speeds and control parameters, for P-controllers with acceleration feedback.

$$
K_{\mathrm{p}}=K_{\mathrm{pL}}=K_{\mathrm{pR}}=0 \cdots 10^{6} \mathrm{~kg} / \mathrm{s}
$$

The control parameters are here integrated into the controlled damping matrix $\boldsymbol{D}_{\mathrm{KP}}$. The related bearing housing vibration velocities are pictured in Figure 11. 


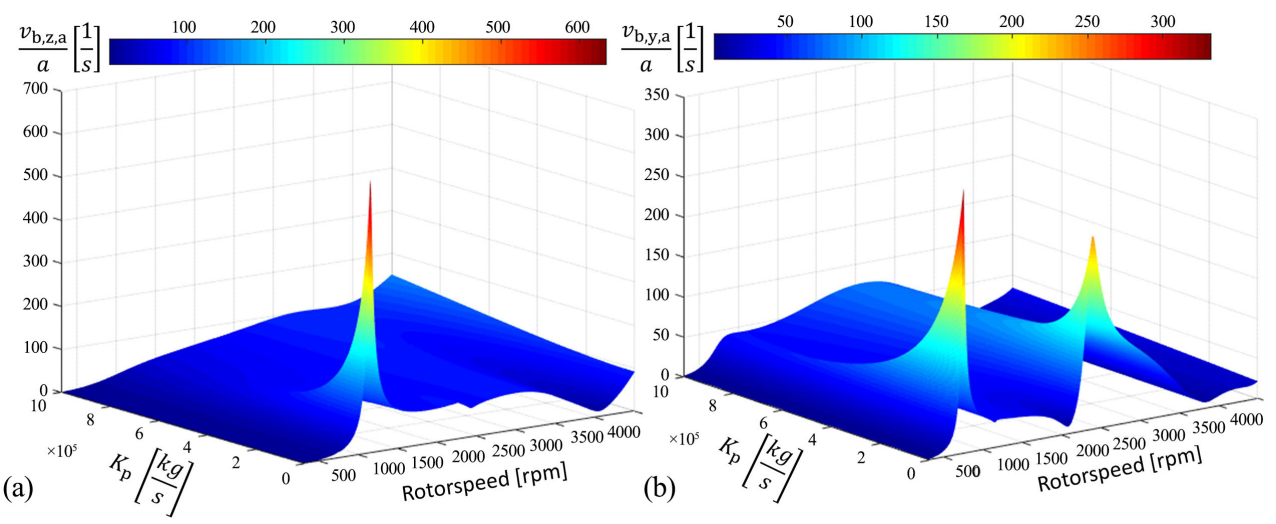

Figure 11. Vertical (a) and horizontal (b) bearing housing vibration velocities, related on bent rotor deflection, for different rotor speeds and control parameters, for P-controllers with velocity feedback.

Figure 11 and Figure 12 show that-contrarily to Section 4.2 and 4.3, where the resonances have been shifted-the resonances remain nearly at the same position. However, the amplitudes in the resonances can be clearly reduced.

The vertical bearing housing vibrations can be reduced in the resonance at $1140 \mathrm{rpm}$ by about $90 \%$ (Figure 11(a)), and the horizontal bearing housing vibrations can be reduced by about $85 \%$ in the resonance at $745 \mathrm{rpm}$ and by about $70 \%$ in the resonance at $2390 \mathrm{rpm}$.

Figure 12 shows, that again for high rotor speeds in combination with large values of $K_{\mathrm{p}}$, the vertical foundation vibrations get very high. But it can be shown, that the amplitudes in the resonances can again be reduced clearly. For example the horizontal vibration in the resonance at $2390 \mathrm{rpm}$ can be reduced by about $80 \%$.

Figure 13 shows, that regarding the actuator forces no resonances are obvious, but for high rotor speeds in combination with large values of $K_{\mathrm{p}}$, also very high actuator forces occur, which is explicable because of the high vertical foundation vibrations, which are shown in Figure 12(a) and Figure 12(b).

\subsection{Discussion of the Results}

When comparing the results form Section 4.2, 4.3 and 4.4, it can be stated that when using a controlled stiffness matrix $\boldsymbol{C}_{\mathrm{KP}}$ (Section 4.2) or a controlled mass matrix $\boldsymbol{M}_{\mathrm{KP}}$ (Section 4.3) or a controlled damping matrix $\boldsymbol{D}_{\mathrm{KP}}$ (section 4.4), the forced vibrations can be clearly influenced.

When using a controlled stiffness matrix $\boldsymbol{C}_{\mathrm{KP}}$, the control parameters are integrated into $\boldsymbol{C}_{\mathrm{KP}}$. With increasing the control parameters, additional stiffness is added to the system. This leads to the fact, that the resonances are shifted to higher rotor speeds, which can clearly be seen in Figure 5 and Figure 6. If negative control parameter would be used, the stiffness of the system would get reduced, and the resonances would be shifted to lower rotor speeds.

However, if a controlled mass matrix $\boldsymbol{M}_{\mathrm{KP}}$ (Section 4.3) is used, the control parameters are now integrated into $\boldsymbol{M}_{\mathrm{KP}}$. When increasing now the control 

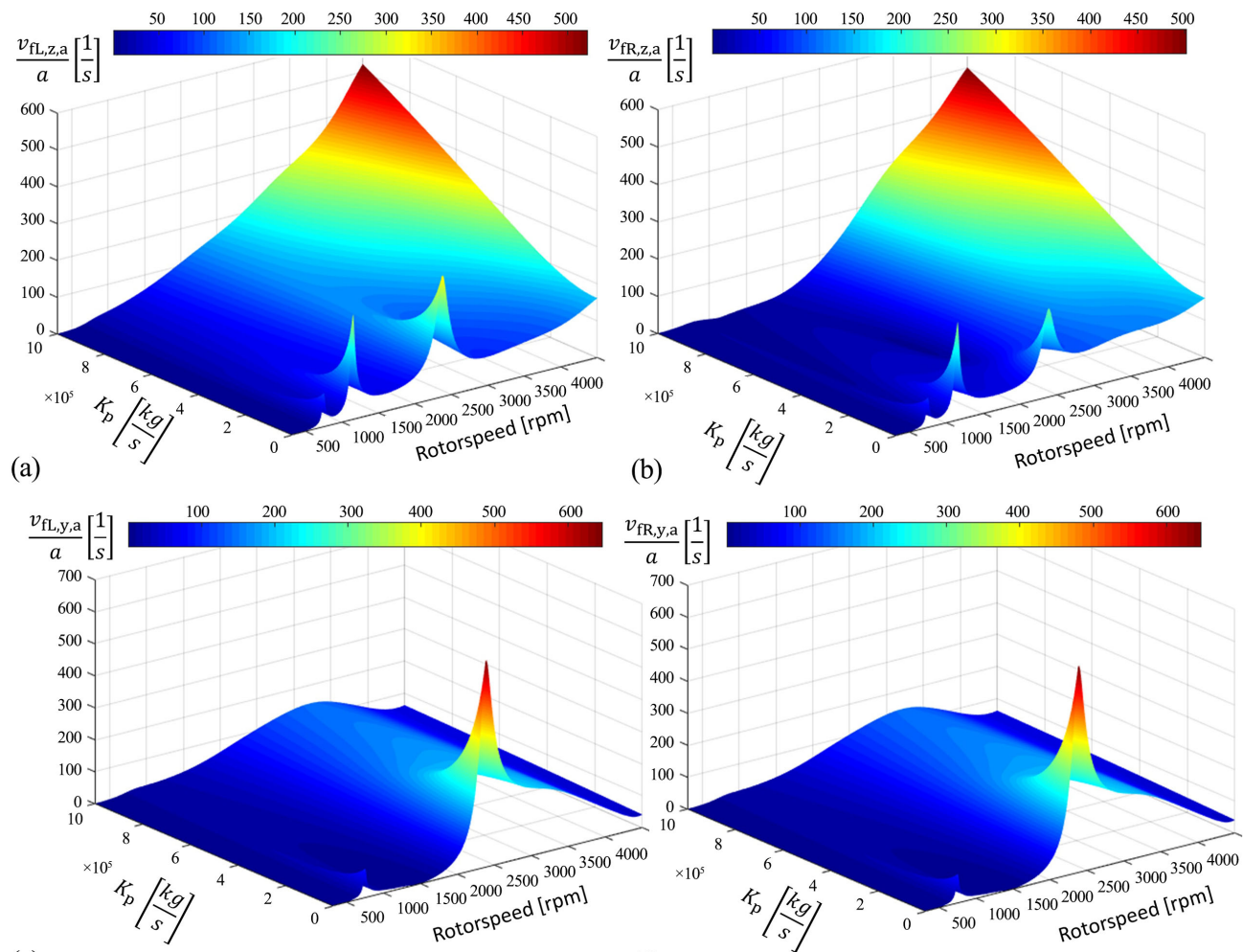

(c)

(d)

Figure 12. Vertical left side (a) and vertical right side (b) foundation vibration velocities and horizontal left side (c) and horizontal right side (d) foundation vibration velocities, related on bent rotor deflection, for different rotor speeds and control parameters, for P-controllers with velocity feedback.

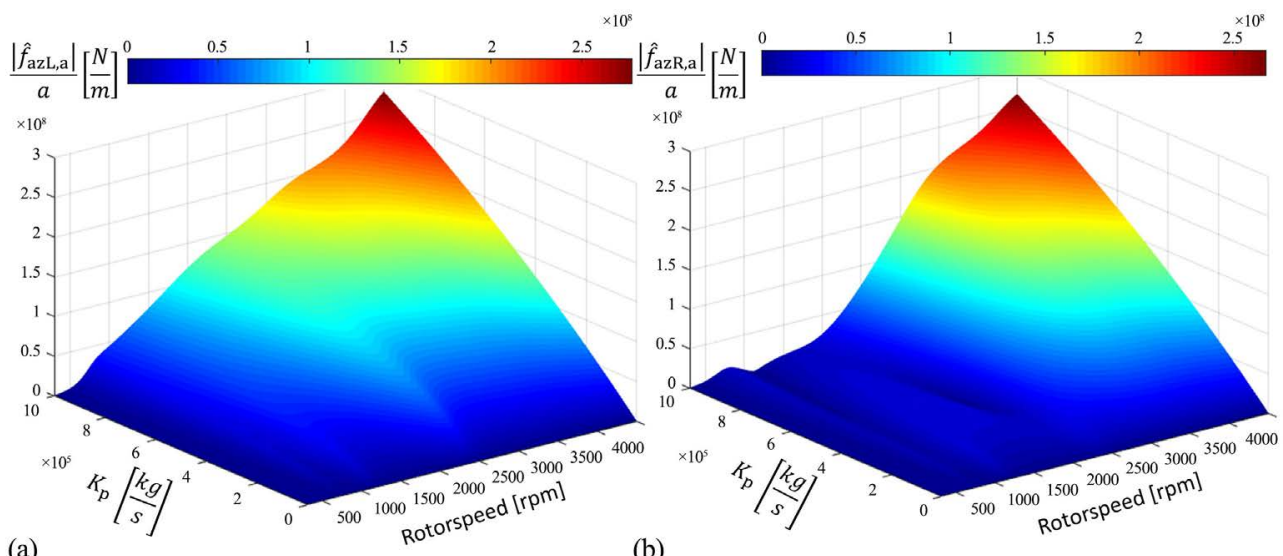

(a)

Figure 13. Absolute values of the vertical actuator forces (a) left side and (b) right side, related on bent rotor deflection, for different rotor speeds and control parameters, for P-controllers with velocity feedback.

parameters, additional mass is added to the system, and the resonances are shifted to lower speeds (Figure 8 and Figure 9). If negative control parameter would be used, the mass of the system would get reduced, and the resonances would be shifted to higher rotor speeds.

If a controlled damping matrix $\boldsymbol{D}_{\mathrm{KP}}$ is used (section 4.4), the control para- 
meters are now added into $\boldsymbol{D}_{\mathrm{KP}}$. When now increasing the control parameters, additional damping is added to the system. The resonances are hardly shifted any more, but the amplitudes in the resonances are clearly reduced (Figure 11 and Figure 12). If negative control parameters would be used, the damping of the system would get reduced, which may mostly not be useful.

Therefore, the most effective measure seems here to use a controlled damping matrix $\boldsymbol{D}_{\mathrm{KP}}$, of course with positive control parameters. If the reduction in the resonances is not sufficient enough, an additional controlled stiffness matrix $\boldsymbol{C}_{\mathrm{KP}}$, or a controlled mass matrix $\boldsymbol{M}_{\mathrm{KP}}$, may be used, to shift the resonances, so that the resonances are shifted to the rotor speeds, where the motor is just not operating. For this strategy the control parameters have to be dependent on the rotor speed, or the controllers are switched off or on for certain rotor speeds.

\section{Conclusion}

The paper presents a theoretical analysis of different vibration control strategies of soft mounted induction motors with sleeve bearings, using active motor foot mounts. After the vibration model has been described, different controllers in combination with different feedback strategies have been mathematically investigated. It could be shown, on which position the control parameters are inserted into the system matrices, depending on the control strategy. The focus of the paper is the analysis of the forced vibrations, caused by dynamic rotor eccentricity-rotor mass eccentricity, magnetic eccentricity and bent rotor deflection. After the mathematically coherences have been described, a numerical example was shown, where the forced vibrations caused by bent rotor deflection have been investigated, for different control strategies. The influence of the controlled stiffness matrix, the controlled mass matrix and the controlled damping matrix on the forced vibrations could be clearly shown in the example. Additionally the actuator forces could be calculated, which is very important, for the dimensioning of the actuators.

\section{Conflicts of Interest}

The author declares no conflicts of interest regarding the publication of this paper.

\section{References}

[1] IEC 60034-14 (International Electrotechnical Commission) (2007) Rotating Electrical Machines Part 14: Mechanical Vibration of Certain Machines with Shaft Heights $56 \mathrm{~mm}$ and Higher-Measurement, Evaluation and Limits of Vibration Severity. 6-10.

[2] Vance, J.M., Zeidan, F.J. and Murphy, B. (2010) Machinery Vibration and Rotordynamics. John Wiley \& Sons, Hoboken, 71-118.

https://doi.org/10.1002/9780470903704.ch3

[3] Friswell, M.I., Penny, J.E.T., Garvey, S.D. and Lees, A.W. (2010) Dynamics of Rotating Machines. Cambridge University Press, Cambridge, 228-291. 
https://doi.org/10.1017/CBO9780511780509

[4] Gasch, R., Nordmann, R. and Pfützner, H. (2002) Rotordynamik. Springer, Berlin-Heidelberg, 17-307.

[5] Rao, J.S. (1996) Rotor Dynamics. John Wiley \& Sons, New York, 69-312.

[6] Preumont, A. (2011) Vibration Control of Active Structures: An Introduction. Springer, Berlin, 41-357. https://doi.org/10.1007/978-94-007-2033-6_3

[7] Janschek, K. (2012) Mechatronic Systems Design: Methods, Models, Concepts. Springer, Berlin, 277-724. https://doi.org/10.1007/978-3-642-17531-2_5

[8] Fuller, C.R., Elliot, S.J. and Nelson, P.A. (1996) Active Control of Vibration. Academic Press, Cambridge, 59-217. https://doi.org/10.1016/B978-012269440-0/50003-0

[9] Ehmann, C. and Nordmann, R. (2012) Comparison of Control Strategies for Active Vibration Control of Flexible Structures. Archives of Control Sciences, 13, 303-312.

[10] Skricka, N. and Markert, R. (2002) Improvements of the Integration of Active Magnetic Bearings. Mechatronics, 12, 1059-1068. https://doi.org/10.1016/S0957-4158(02)00013-2

[11] Schweitzer, G. and Maslen, E.H. (2009) Magnetic Bearings. Springer, Berlin, 27-433.

[12] Noshadi, A., Shi, J., Lee, W.S., Shi, P. and Kalam, A. (2017) Robust Control of an Active Magnetic Bearing System Using $\mathrm{H} \infty$ and Disturbance Observer-Based Control. Journal of Vibration and Control, 23, 1857-1870. https://doi.org/10.1177/1077546315602421

[13] Anantachaisilp, P. and Lin, Z. (2017) Fractional Order PID Control of Rotor Suspension by Active Magnetic Bearings. Actuators, 6, 1-31. https://doi.org/10.3390/act6010004

[14] Ran, S., Hu, Y. and Wu, H. (2018) Design, Modeling, and Robust Control of the Flexible Rotor to Pass the First Bending Critical Speed with Active Magnetic Bearing. Advances in Mechanical Engineering, 10, 1-13.

[15] Ushijima, T. and Kumakawa, S. (1993) Active Engine Mount with Piezo-Actuator for Vibration Control. SAE Technical Paper 930201.

[16] Ulbrich, H. (1994) A Comparison of Different Actuator Concepts for Applications in Rotating Machinery. International Journal of Rotating Machinery, 1, 61-71. https://doi.org/10.1155/S1023621X94000060

[17] Chen, H.M., Lewis, P., Donald, S. and Wilson, S. (1998) Active Mounts. Journal of the Acoustical Society of America, 91, 2301.

[18] Sui, L., Xiong, X. and Shi, G. (2012) Piezoelectric Actuator Design and Application on Active Vibration Control. Physics Procedia, 25, 1388-1396. https://doi.org/10.1016/j.phpro.2012.03.251

[19] Sun, W., Gao, H. and Yao, B. (2013) Adaptive Robust Vibration Control of Full-Car Active Suspensions with Electrohydraulic Actuators. IEEE Transactions on Control Systems Technology, 21, 2417-2422. https://doi.org/10.1109/TCST.2012.2237174

[20] Werner, U. (2018) Vibration Control of Soft Mounted Induction Motors with Sleeve Bearings Using Active Motor Foot Mounts: A Theoretical Analysis. Archive of Applied Mechanics, 88, 1657-1682. https://doi.org/10.1007/s00419-018-1393-7

[21] Früchtenicht, J., Jordan, H. and Seinsch, H.O. (1982) Exzentrizitätsfelder als Ursache von Laufinstabilitäten bei Asynchronmaschinen Archiv für Elektrotechnik Bd. 65 Teil 1, 271-281, Teil 2, 283-292.

[22] Belmans, R., Vandenput, A. and Geysen, W. (1987) Calculation of the Flux Density 
and the Unbalanced Pull in Two Pole Induction Machines. Electrical Engineering, 70, 151-161.

[23] Seinsch, H.O. (1992) Oberfelderscheinungen in Drehfeldmaschinen. Teubner, Stuttgart, 31-109.

[24] Belmans, R., Vandenput, A. and Geysen, W. (1987) Influence of Unbalanced Magnetic Pull on the Radial Stability of Flexible-Shaft Induction Machines. IEE Proceedings-B Electric Power Applications, 134, 101-109. https://doi.org/10.1049/ip-b.1987.0013

[25] Arkkio, A., Antila, M., Pokki, K., Simon, A. and Lantto, E. (2000) Electromagnetic Force on a Whirling Cage Rotor. IEE Proceedings-Electric Power Applications, 147, 353-360. https://doi.org/10.1049/ip-epa:20000523

[26] Holopainen, T.P. (2004) Electromechanical Interaction in Rotor Dynamics of Cage Induction Motors. VTT Technical Research Centre of Finland, PhD Thesis, Helsinki University of Technology, 14-54.

[27] Dorrell, D.G. (2011) Sources and Characteristics of Unbalanced Magnetic Pull in Three-Phase Cage Induction Motors with Axial-Varying Rotor Eccentricity. IEEE Transactions on Industry Applications, 47, 12-24. https://doi.org/10.1109/TIA.2010.2090845

[28] Werner, U. (2017) Mathematical Multibody Model of a Soft Mounted Induction Motor Regarding Forced Vibrations Due to Dynamic Rotor Eccentricities Considering Electromagnetic Field Damping. Journal of Applied Mathematics and Physics, 5, 346-364. https://doi.org/10.4236/jamp.2017.52032

[29] Tondl, A. (1965) Some Problems of Rotor Dynamics. Chapman \& Hall, London, $1-434$.

[30] Lund, J. and Thomsen, K. (1978) A Calculation Method and Data for the Dynamics of Oil Lubricated Journal Bearings in Fluid Film Bearings and Rotor Bearings System Design and Optimization. ASME, New York, 1-28.

[31] Hori, Y. (2006) Hydrodynamic Lubrication. Springer, Berlin, 23-46.

[32] Someja, T. (2013) Journal Bearing Databook. Springer, Berlin, 1-294. 